\title{
Cyclic Homology and Quantum Orbits
}

\author{
Tomasz MASZCZYK and Serkan SÜTL $\ddot{U}$ \\ Institute of Mathematics, University of Warsaw, Warsaw, Poland \\ E-mail: t.maszczyk@uw.edu.pl,serkansutlu@gmail.com
}

Received December 07, 2014, in final form May 12, 2015; Published online May 30, 2015

http://dx.doi.org/10.3842/SIGMA.2015.041

\begin{abstract}
A natural isomorphism between the cyclic object computing the relative cyclic homology of a homogeneous quotient-coalgebra-Galois extension, and the cyclic object computing the cyclic homology of a Galois coalgebra with SAYD coefficients is presented. The isomorphism can be viewed as the cyclic-homological counterpart of the Takeuchi-Galois correspondence between the left coideal subalgebras and the quotient right module coalgebras of a Hopf algebra. A spectral sequence generalizing the classical computation of Hochschild homology of a Hopf algebra to the case of arbitrary homogeneous quotient-coalgebra-Galois extensions is constructed. A Pontryagin type self-duality of the Takeuchi-Galois correspondence is combined with the cyclic duality of Connes in order to obtain dual results on the invariant cyclic homology, with SAYD coefficients, of algebras of invariants in homogeneous quotient-coalgebra-Galois extensions. The relation of this dual result with the Chern character, Frobenius reciprocity, and inertia phenomena in the local Langlands program, the Chen-Ruan-Brylinski-Nistor orbifold cohomology and the Clifford theory is discussed.
\end{abstract}

Key words: cyclic homology; homogenous quotient-coalgebra-Galois extensions; TakeuchiGalois correspondence; Pontryagin duality

2010 Mathematics Subject Classification: 19D55; 57T15; 06A15; 46A20

\section{Introduction}

It is well known due to Takeuchi [39] (vastly extended by van Oystaeyen-Zhang [40] and Schauenburg [37]) that given a Hopf algebra $\mathcal{H}$ there is a Galois 1-1 correspondence between its left coideal subalgebras, for which $\mathcal{H}$ is a faithfully flat algebra extension, and its quotient right module coalgebras, for which $\mathcal{H}$ is a faithfully coflat coalgebra coextension.

Such a correspondence can also be viewed as a relation between the extensions of comodule algebras and the coextensions of module coalgebras, both of which having their specific homological invariants (Hochschild, cyclic, periodic cyclic and negative cyclic homology) computed from appropriate cyclic objects.

For algebra extensions, it is a relative cyclic object introduced by Kadison [28], and for comodule algebras it is a cyclic object with stable anti-Yetter-Drinfeld (SAYD) coefficients introduced by Hajac-Khalkhali-Rangipour-Sommerhäuser [24, 25], and independently by JaraŞtefan [27] (in a cyclic dual version).

For module coalgebras, it is a cyclic object with SAYD coefficients which is cyclic dual to that of [24], and for coalgebra extensions it is the cyclic dual of the Pontryagin dual analogue of $[28]$.

Therefore it is very natural to ask whether these different types of cyclic objects are related in the context of the aforementioned Takeuchi-Galois correspondence.

This question goes far beyond the Galois theory (herein the theory of so called homogeneous quotient coalgebra-Galois extensions [11]) and reaches topology. In the case of smooth functions on compact Lie groups and their homogeneous spaces, relative periodic cyclic homology computes the vector bundle of de Rham cohomology of the stabilizer over the homogeneous 
space. This bundle is equipped with the Gauss-Manin connection (see [21] for noncommutative fibrations with commutative base) determining a local system of coefficients whose cohomology appears in the second page of the Leray spectral sequence interpolating between the cohomology of the compact Lie group and the cohomology of the homogeneous space. Then the above Takeuchi-Galois correspondence boils down to one between stabilizers and orbits, and the algebra extension describes the orbital map.

Moreover, the cyclic object, with SAYD coefficients, of a module coalgebra is cyclic dual to the cocyclic object generalizing the one used by Connes-Moscovici in the proof of a generalized transversal local index theorem for foliated spaces, where the index computation relies on a symmetry governed by a Hopf algebra [16, 17, 24].

The aim of the present paper is to prove that these two types of homological invariants are isomorphic in a natural way. More explicitly,

Theorem 3.4. Let $\mathcal{I} \subseteq \mathcal{H}$ be a coideal right ideal in a Hopf algebra $\mathcal{H}$ such that $\mathcal{H}^{\mathrm{co}} \mathcal{H} / \mathcal{I} \subseteq \mathcal{H}$ is an $\mathcal{H} / \mathcal{I}$-Galois extension. Let also $\operatorname{ad}(\mathcal{H})=\mathcal{H}$ be the left-right $S A Y D$ module with the right adjoint action, and the left coaction given by the comultiplication of $\mathcal{H}$. Then there exists an isomorphism

$$
\psi_{n}: \mathrm{C}_{n}(\mathcal{H} / \mathcal{I}, \operatorname{ad}(\mathcal{H}))_{\mathcal{H}} \longrightarrow \mathrm{C}_{n}\left(\mathcal{H} \mid \mathcal{H}^{\operatorname{co} \mathcal{H} / \mathcal{I}}\right)
$$

of cyclic modules, defined by (3.6), (3.7).

Since the domain of the isomorphism depends only on the right $\mathcal{H}$-module quotient coalgebra of $\mathcal{H}$, and the codomain depends on the left comodule subalgebra of $\mathcal{H}$, this isomorphism can be viewed as a cyclic-homological Takeuchi-Galois transform accompanying the Takeuchi-Galois transform $\mathcal{H} / \mathcal{I} \mapsto \mathcal{H}^{\text {co } \mathcal{H} / \mathcal{I}}$.

This perspective manifested itself in the paper of Jara-Ştefan [27], where the first example of the cyclic homology with SAYD coefficients different from the modular pairs in involution of Connes-Moscovici was presented. Their main result [27, Theorem 3.7] is an isomorphism of cyclic objects computing relative cyclic homology of a Hopf-Galois extension, and cyclic homology of the Galois Hopf algebra itself with an appropriate SAYD coefficients (defined via the Miyashita-Ulbrich action of the Hopf algebra).

However, the Hopf-Galois context is too narrow to cover the full context of the TakeuchiGalois correspondence (making sense only for a restricted correspondence between left comodule subalgebras $\mathcal{B} \subseteq \mathcal{H}$ such that $\mathcal{B}^{+} \mathcal{H}$ is a Hopf ideal and quotient Hopf-algebras [39]) and misses important examples such as non-standard Podleś quantum spheres. In such cases, instead of a Hopf algebra of Galois symmetry one has merely a coaugmented quotient coalgebra of a bigger Hopf-algebra acting transitively on both the base and the total quantum space of a quantum principal bundle (so called homogeneous quotient-coalgebra-Galois extension). Then a Miyashita-Ulbrich type action doesn't make sense, and the Jara-Ştefan isomorphism [27, Theorem 3.7] cannot be applied.

Nevertheless, as we show below, when restricted to the homogeneous Hopf-quotient-Galois case our isomorphism and that of Jara-Ştefan [27, Theorem 3.7], despite the apparently different definitions, essentially coincide.

A deeper conceptual motivation of the coalgebra-Galois approach, beyond the context of the Takeuchi-Galois correspondence, comes from the so called coisotropic creed of Poisson geometry [32]. The latter, accepted to bypass the problem of poverty of Poisson subgroups of Poisson groups, launched the theory of coisotropic subgroups. The quantized counterpart of coisotropic subgroups of a Poisson group is a quotient coaugmented right module coalgebra of a Hopf algebra by a coideal right ideal. The non-standard Podleś quantum spheres are examples of quantum orbits corresponding to such coalgebraic quantum stabilizers [18].

It is worth noticing that even in such a simple homogeneous $\mathcal{H}$-Galois extension as $k=$ $\mathcal{H}^{\text {co } \mathcal{H}} \rightarrow \mathcal{H}$, computing the relative Hochschild homology (being the Hochschild homology of 
the Hopf algebra itself) is nontrivial and of fundamental interest [3, 6, 14, 20, 22, 23]. The following folklore result, proven explicitly first by Feng-Tsygan [20], next by Bichon [3] by a similar method with a different perspective, but in fact already implicitly contained in CartanEilenberg's book (it is enough to dualize a consequence of [12, Theorem VIII.3.1] and rephrase the result in terms of Hopf algebras and Hopf algebra homology) says that

Corollary 3.6. For any Hopf algebra $\mathcal{H}$,

$$
\mathrm{HH}_{\bullet}(\mathcal{H})=\operatorname{Tor}_{\bullet}^{\mathcal{H}}(k, \operatorname{ad}(\mathcal{H})),
$$

where on the right hand side the left $\mathcal{H}$-module structure on $\mathcal{H}$ comes from its canonical SAYD module structure.

We generalize this result to the case of arbitrary homogeneous quotient coalgebra-Galois extensions as follows

Theorem 3.5. Let $\mathcal{I} \subseteq \mathcal{H}$ be a coideal right ideal in a Hopf algebra $\mathcal{H}$ such that $\mathcal{H}^{\text {co } \mathcal{H} / \mathcal{I}} \subseteq \mathcal{H}$ be a homogeneous $\mathcal{H} / \mathcal{I}$-Galois extension. Then there exists a spectral sequence (constructed in the proof) such that

$$
\mathrm{HH}_{\bullet}\left(\mathcal{H} \mid \mathcal{H}^{\mathrm{co} \mathcal{H} / \mathcal{I}}\right)=\mathrm{E}_{\bullet}^{2}, 0, \quad \mathrm{E}_{\bullet, \bullet}^{2} \Longrightarrow \operatorname{Tor}_{\bullet}^{\mathcal{H}}(k, \operatorname{ad}(\mathcal{H}))
$$

In particular, we have a five-term exact sequence

$$
\begin{aligned}
\operatorname{Tor}_{2}^{\mathcal{H}}(k, \operatorname{ad}(\mathcal{H})) & \rightarrow \mathrm{HH}_{2}\left(\mathcal{H} \mid \mathcal{H}^{\mathrm{co} \mathcal{H} / \mathcal{I}}\right) \rightarrow \mathrm{H}_{0}\left(\operatorname{Tor}_{1}^{\mathcal{H}}\left((\mathcal{H} / \mathcal{I})^{\otimes \bullet+1}, \operatorname{ad}(\mathcal{H})\right)\right) \rightarrow \cdots \\
\cdots \rightarrow \operatorname{Tor}_{1}^{\mathcal{H}}(k, \operatorname{ad}(\mathcal{H})) & \rightarrow \mathrm{HH}_{1}\left(\mathcal{A} \mid \mathcal{H}^{\mathrm{co} \mathcal{H} / \mathcal{I}}\right) \rightarrow 0 .
\end{aligned}
$$

The classical result follows from this spectral sequence by the degeneration argument.

Furthermore, inverting arrows in all diagrams defining the cyclic-homological TakeuchiGalois transform, interchanging everywhere left and right, next applying Connes' cyclic duality and finally inverting the resulting isomorphism we obtain

Theorem 3.7. Let $\mathcal{B} \subseteq \mathcal{H}$ be a left comodule subalgebra in a Hopf algebra $\mathcal{H}$ such that $\mathcal{H} \rightarrow$ $\mathcal{H} / \mathcal{B}^{+} \mathcal{H}$ is a $\mathcal{B}-$ Galois coextension, and $\operatorname{coad}(\mathcal{H})=\mathcal{H}$ be the right-left $S A Y D$ module with the right action given by the multiplication of $\mathcal{H}$, and the left coadjoint coaction. Then there exists an isomorphism

$$
\gamma_{n}: \mathrm{C}_{n}(\mathcal{B}, \operatorname{coad}(\mathcal{H}))^{\mathcal{H}} \longrightarrow \mathrm{C}_{n}\left(\mathcal{H} \mid \mathcal{H} / \mathcal{B}^{+} \mathcal{H}\right)
$$

of cyclic modules, defined by (3.13), (3.14).

Since the domain of the isomorphism depends only on the left comodule subalgebra $\mathcal{B}$ of a Hopf algebra $\mathcal{H}$, and the codomain depends on the right $\mathcal{H}$-module quotient coalgebra $\mathcal{H} / \mathcal{B}^{+} \mathcal{H}$ of $\mathcal{H}$, it can be viewed as a cyclic-homological dual Takeuchi-Galois transform accompanying the Takeuchi-Galois transform $\mathcal{B} \mapsto \mathcal{H} / \mathcal{B}^{+} \mathcal{H}$.

Surprisingly, this dual picture is even more interesting than the original construction directly motivated by classical geometry. The point is that it makes sense in classical geometry as well, but then is related to quite nontrivial phenomena connecting geometry and representation theory. In Subsection 3.5.2 we discuss relations of the dual picture to the Chern character, the Frobenius reciprocity and inertia phenomena in the local Langlands program, the Chen-RuanBrylinski-Nistor orbifold cohomology and the Clifford theory.

\section{Preliminaries}

In this section we recall the material we will use in the sequel. In the first subsection we recall the coalgebra-Galois extensions, as well as the algebra-Galois coextensions. In the second 
subsection we discuss the Pontryagin like duality between discrete and linearly compact vector spaces. Upon recalling the Takeuchi-Galois correspondence between left comodule subalgebras and right module quotient coalgebras of a Hopf algebra, we show Pontryagin self-duality of this correspondence. In the third subsection we recall the relative cyclic homology of algebra extensions. Finally, the Hopf-cyclic homology, with coefficients, of coalgebras is recalled in the fourth subsection.

Throughout the paper, all algebras, coalgebras, and Hopf algebras are over a field $k$, and similarly all unadorned tensor product symbols $\otimes$ are also over $k$. Suppressing the summation, we will write $\Delta(c)=c_{(1)} \otimes c_{(2)}$ for a comultiplication, $\rho(v)=v_{\langle 0\rangle} \otimes v_{\langle 1\rangle}$ for a right coaction, and $\lambda(v)=v_{\langle-1\rangle} \otimes v_{\langle 0\rangle}$ for a left coaction.

\subsection{Coalgebra-Galois extensions and algebra-Galois coextensions}

In this subsection we recall the definition and basic properties of the coalgebra-Galois extensions, and the algebra-Galois coextensions from $[10,11]$. Fix the base field $k$.

We begin with the coalgebra-Galois extensions. Let $\mathcal{C}$ be a coalgebra coaugmented by the choice of a group-like element $e \in \mathcal{C}$. We say that an algebra $\mathcal{A}$ is a $\mathcal{C}$-algebra if there is given a right entwining $\psi: \mathcal{C} \otimes \mathcal{A} \rightarrow \mathcal{A} \otimes \mathcal{C}$. Then $\mathcal{A}$ becomes a right $\mathcal{C}$-comodule via $\rho: \mathcal{A} \rightarrow \mathcal{A} \otimes \mathcal{C}$ defined as $\rho(a)=a_{\langle 0\rangle} \otimes a_{\langle 1\rangle}:=\psi(e \otimes a)$. Then the subspace $\mathcal{A}^{\text {co } \mathcal{C}}$ of coaction invariants of $\mathcal{A}$ defined as

$$
\mathcal{A}^{\mathrm{co} \mathcal{C}}:=\{b \in \mathcal{A} \mid \psi(e \otimes b)=b \otimes e\}
$$

is a subalgebra of $\mathcal{A}$. Note that $\mathcal{A}^{\text {coC }}$ can be defined as a limit of a diagram in vector spaces

$$
\mathcal{A}^{\operatorname{co} \mathcal{C}}=\operatorname{Eq}(\mathcal{A} \rightrightarrows \mathcal{A} \otimes \mathcal{C})
$$

consisting of a pair of maps $a \mapsto a_{\langle 0\rangle} \otimes a_{\langle 1\rangle}$ and $a \mapsto a \otimes e$.

An algebra extension $\mathcal{B} \rightarrow \mathcal{A}$ is called a $\mathcal{C}$-extension if $\mathcal{A}$ is a $\mathcal{C}$-algebra and $\mathcal{B}=\mathcal{A}^{\text {co } \mathcal{C}}$. Finally, a $\mathcal{C}$-extension $\mathcal{B} \rightarrow \mathcal{A}$ is said to be Galois if the left $\mathcal{A}$-linear right $\mathcal{C}$-colinear map

$$
\text { can }: \mathcal{A} \otimes_{\mathcal{B}} \mathcal{A} \longrightarrow \mathcal{A} \otimes \mathcal{C}, \quad a \otimes_{\mathcal{B}} a^{\prime} \mapsto a a_{\langle 0\rangle}^{\prime} \otimes a^{\prime}{ }_{\langle 1\rangle}
$$

is bijective. The map (2.1) is called the canonical map of the $\mathcal{C}$-extension.

A standard example of a $\mathcal{C}$-algebra $\mathcal{A}$ comes from any coaugmented right $\mathcal{H}$-module coalgebra $\mathcal{C}$ and any right $\mathcal{H}$-comodule algebra $\mathcal{A}$ over a Hopf algebra $\mathcal{H}$. If we denote by $a \mapsto a_{[0]} \otimes a_{[1]}$ the right $\mathcal{H}$-coaction on $\mathcal{A}$ the corresponding entwining reads as $\psi(c \otimes a)=a_{[0]} \otimes c \cdot a_{[1]}$.

An interesting, due to the geometric examples it covers, class of standard coalgebra-Galois extensions consists of so called quotient coalgebra-Galois extensions defined as follows. In this setting, one lets $\mathcal{H}$ to be a Hopf algebra, a coaugmented right $\mathcal{H}$-module coalgebra $\mathcal{C}$ a quotient $\mathcal{H} / \mathcal{I}$ of $\mathcal{H}$ by a coideal right ideal $\mathcal{I} \subseteq \mathcal{H}$, and $\mathcal{A}$ a right $\mathcal{H}$-comodule algebra via $\widetilde{\rho}: \mathcal{A} \longrightarrow \mathcal{A} \otimes \mathcal{H}$. Then the composition

$$
\rho: \mathcal{A} \stackrel{\widetilde{\rho}}{\longrightarrow} \mathcal{A} \otimes \mathcal{H} \stackrel{\mathcal{A} \otimes \pi}{\longrightarrow} \mathcal{A} \otimes \mathcal{H} / \mathcal{I}
$$

expresses the standard right $\mathcal{H} / \mathcal{I}$-coaction on $\mathcal{A}$. Therefore the subalgebra of the coaction invariants can be expressed diagrammatically in vector spaces as the equalizer

$$
\mathcal{A}^{\mathrm{co} \mathcal{H} / \mathcal{I}}=\operatorname{Eq}(\mathcal{A} \rightrightarrows \mathcal{A} \otimes \mathcal{H} / \mathcal{I}),
$$

where one arrow is $\rho$ defined by (2.2), and the other is the composition

$$
\mathcal{A} \stackrel{\cong}{\longrightarrow} \otimes k \stackrel{\mathcal{A} \otimes \eta}{\longrightarrow} \mathcal{A} \otimes \mathcal{H} \stackrel{\mathcal{A} \otimes \pi}{\longrightarrow} \mathcal{A} \otimes \mathcal{H} / \mathcal{I}
$$


Finally, a Galois $\mathcal{H} / \mathcal{I}$-extension $\mathcal{A}^{\text {co } \mathcal{H} / \mathcal{I}} \rightarrow \mathcal{A}$ is called a quotient coalgebra-Galois extension. If $\mathcal{I}$ is a Hopf ideal, we call such an extension quotient Hopf-Galois extension.

The quantum instanton bundle of $[4,5]$ is a quotient coalgebra-Galois extension, and it uses the full generality of the quotient coalgebra-Galois extensions as $\mathcal{A} \neq \mathcal{H}$ and $\mathcal{I} \neq 0$.

For $\mathcal{I}=0$, the quotient coalgebra-Galois extensions recover the Hopf-Galois extensions, and in case of $\mathcal{A}=\mathcal{H}$ they are called homogeneous coalgebra-Galois extensions. In particular, viewing $\mathcal{H}$ as a right $\mathcal{H}$-comodule algebra via its comultiplication, one obtains the homogeneous $\mathcal{H} / \mathcal{I}$-Galois extensions

$$
\rho: \mathcal{H} \stackrel{\Delta}{\longrightarrow} \mathcal{H} \otimes \mathcal{H} \stackrel{\mathcal{H} \otimes \pi}{\longrightarrow} \mathcal{H} \otimes \mathcal{H} / \mathcal{I}, \quad h \mapsto h_{(1)} \otimes \overline{h_{(2)}}
$$

In the context of the faithfully flat homogeneous extensions, the diagrammatical definition of invariants of the coaction (2.3) plays an important role in the context of Pontryagin self-duality of the Takeuchi-Galois correspondence considered in the next subsection.

Such Galois extensions correspond to the quantum homogeneous spaces considering $\mathcal{H}=$ $\mathcal{O}(G)$ the algebra of functions on a quantum group $G=\operatorname{Spec}(\mathcal{H})$, and $\mathcal{B}=\mathcal{O}(X)$ the algebra of functions on a quantum space $X=\operatorname{Spec}(\mathcal{B})$. Then the $G$-action on $X$ is encoded by the $\mathcal{H}$ coaction [18, Section 1]. There is a celebrated example of this construction due to Podleś $[8,35]$ which is a quantum spherical fibration $S U_{q}(2) \rightarrow S_{q, \mu, \nu}^{2}$, see [9], a quantum deformation of the classical Hopf fibration of a 3 -sphere over a 2 -sphere into circles.

We now recall the algebra-Galois coextensions. Let $\mathcal{B}$ be an algebra augmented by the choice of a character $y: \mathcal{B} \rightarrow k$. We say that a coalgebra $\mathcal{D}$ is a $\mathcal{B}$-coalgebra if there is given a left entwining $\varphi: \mathcal{B} \otimes \mathcal{D} \rightarrow \mathcal{D} \otimes \mathcal{B}$. Then $\mathcal{D}$ becomes a left $\mathcal{B}$-module via $\ell: \mathcal{B} \otimes \mathcal{D} \rightarrow \mathcal{D}$ defined as $\ell=(\mathcal{D} \otimes y) \circ \varphi$. Then the quotient space $\mathcal{D} / \mathcal{B}^{+} \mathcal{D}$ of action coinvariants of $\mathcal{D}$ defined by

$$
\mathcal{B}^{+}:=\{b \in \mathcal{B} \mid y(b)=0\}
$$

is a quotient coalgebra of $\mathcal{D}$. Note that $\mathcal{D} / \mathcal{B}^{+} \mathcal{D}$ can be defined as a colimit of a diagram in vector spaces

$$
\mathcal{D} / \mathcal{B}^{+} \mathcal{D}=\operatorname{Coeq}(\mathcal{D} \leftleftarrows \mathcal{B} \otimes \mathcal{D})
$$

consisting of a pair of maps $\ell: b \otimes d \mapsto b \cdot d$ and $b \otimes d \mapsto y(b) d$.

A coalgebra coextension $\mathcal{D} \rightarrow \mathcal{C}$ is called a $\mathcal{B}$-coextension if $\mathcal{D}$ is a $\mathcal{B}$-coalgebra and $\mathcal{C}=$ $\mathcal{D} / \mathcal{B}^{+} \mathcal{D}$. Finally, a $\mathcal{B}$-coextension $\mathcal{D} \rightarrow \mathcal{C}$ is said to be Galois if the left $\mathcal{B}$-module, right $\mathcal{D}$ comodule map

$$
\text { cocan }: \mathcal{B} \otimes \mathcal{D} \longrightarrow \mathcal{D} \square_{\mathcal{C}} \mathcal{D}, \quad b \otimes d \mapsto b \cdot d_{(1)} \otimes d_{(2)}
$$

is bijective. The map (2.4) is called the cocanonical map of the coalgebra $\mathcal{B}$-coextension.

It is evident that the notion of algebra-Galois coalgebra coextension dualizes the notion of coalgebra-Galois algebra extension by formal inverting all arrows in all diagrams and interchanging left and right. In the Hopf-Galois setting the construction dualizes the Hopf-Galois extensions [38].

\subsection{Formal Pontryagin duality and Takeuchi-Galois correspondence}

In this subsection we will first summarize the basic properties of the dualization functor on vector spaces from [2]. We will then recall a one-to-one correspondence between the coideal subalgebras and quotient coalgebras, known as the Takeuchi-Galois correspondence [39].

From the point of view of linear topological vector spaces with continuous linear mappings as morphisms, the dualization functor defines an equivalence between the opposite category of 
discrete vector spaces and the category of linearly compact vector spaces [2, Proposition 24.8]. Moreover, it transforms naturally the algebraic tensor product of discrete vector spaces into a completed one of linearly compact spaces [2, Corollary 24.25]. In other words, dualization is a strong monoidal functor. Therefore one can regard on equal footing all structures defined by diagrams in vector spaces together with their dual counterparts obtained by reversing all arrows in all necessary diagrams. This regards linear subspaces and quotient spaces, (co)algebras and Hopf algebras, as well as their bi(co)modules or one sided (co)modules, one-sided and two-sided (co)ideals, and their (co)tensor products. Hence, having a diagrammatical proof of a theorem in a symmetric monoidal category of linear (topological) spaces, one has automatically a dual theorem after an appropriate dualization of the structures. It is well known that the notion of Hopf algebra is self-dual. In particular, it transforms the Hopf group-algebra of a discrete group into a linearly compact topological Hopf algebra of functions on that group, customarily regarded as a group algebra of a dual compact quantum group. Therefore we will call this duality Pontryagin to separate it from cyclic duality. We will show that the notion of SAYD module is Pontryagin self-dual up to the interchange of left and right. According to $[9,10]$ the notion of coalgebra-Galois extension of algebras is Pontryagin dual to the notion of algebra-Galois coextension of coalgebras. Both dualities play a role in the present paper.

For any Hopf algebra $\mathcal{H}$ with multiplication $\mu: \mathcal{H} \otimes \mathcal{H} \longrightarrow \mathcal{H}$, unit $\eta: k \longrightarrow \mathcal{H}$, comultiplication $\Delta: \mathcal{H} \longrightarrow \mathcal{H} \otimes \mathcal{H}$, counit $\varepsilon: \mathcal{H} \longrightarrow k$, and antipode $S: \mathcal{H} \longrightarrow \mathcal{H}$, Takeuchi introduces in [39] the one-to-one (Galois) correspondence

$\{\mathcal{B} \subseteq \mathcal{H} \mid \mathcal{B}$ is a left coideal subalgebra, $\mathcal{H}$ is faithfully flat over $\mathcal{B}\}$

$\uparrow \downarrow$

$\{\mathcal{I} \subseteq \mathcal{H} \mid \mathcal{I}$ is a coideal right ideal, $\mathcal{H}$ is faithfully coflat over $\mathcal{H} / \mathcal{I}\}$

under which $\mathcal{B} \mapsto \mathcal{B}^{+} \mathcal{H}$ and $\mathcal{I} \mapsto \mathcal{H}^{\text {co } \mathcal{H} / \mathcal{I}}$.

A crucial observation for our purpose is that the above correspondence can be written categorically as an equivalence between the category of left $\mathcal{H}$-comodule flat extensions $i: \mathcal{B} \rightarrow \mathcal{H}$ and the category of right $\mathcal{H}$-module coflat coextensions $\pi: \mathcal{H} \rightarrow \mathcal{C}$, which is given by

$$
\mathcal{B} \mapsto \operatorname{Coeq}(\mathcal{H} \leftleftarrows \mathcal{B} \otimes \mathcal{H}), \quad \mathcal{C} \mapsto \operatorname{Eq}(\mathcal{H} \rightrightarrows \mathcal{H} \otimes \mathcal{C})
$$

where the parallel pair of left arrows in the coequalizer and the parallel pair of right arrows in the equalizer read as composites

$$
\begin{array}{ll}
\mathcal{H} \stackrel{\mu}{\longleftarrow} \mathcal{H} \otimes \mathcal{H} \stackrel{\iota \otimes \mathcal{H}}{\longleftarrow} \mathcal{B} \otimes \mathcal{H}, \quad & \mathcal{H} \stackrel{\Delta}{\longrightarrow} \mathcal{H} \otimes \mathcal{H} \stackrel{\mathcal{H} \otimes \pi}{\longrightarrow} \mathcal{H} \otimes \mathcal{C}, \\
\mathcal{H} \stackrel{\cong}{\longleftarrow} \otimes \mathcal{H} \stackrel{\varepsilon \iota \otimes \mathcal{H}}{\longleftarrow} \mathcal{B} \otimes \mathcal{H}, \quad & \mathcal{H} \stackrel{\cong}{\longrightarrow} \otimes k \stackrel{\mathcal{H} \otimes \pi \eta}{\longrightarrow} \mathcal{H} \otimes \mathcal{C},
\end{array}
$$

respectively. It is thus evident that this correspondence is Pontryagin self-dual up to an interchange of left and right in dual structures. We will say that $\mathcal{B}$ and $\mathcal{C}$ as above are Takeuchi-Galois transforms of each other.

\subsection{Relative cyclic homology of (co)algebra (co)extensions}

We start with a quick detour on the relative cyclic homology of algebra extensions. For any $\mathcal{B}$-bimodule $\mathcal{M}$ we let

$$
[\mathcal{M}]_{\mathcal{B}}:=\mathcal{M} /[\mathcal{M}, \mathcal{B}]
$$

where $[\mathcal{M}, \mathcal{B}]$ is the subspace of $\mathcal{M}$ generated by all commutators $[m, b]:=m \cdot b-b \cdot m$. We will denote by $[m]_{\mathcal{B}}$ the class of $m \in \mathcal{M}$ in $[\mathcal{M}]_{\mathcal{B}}$. 
The relative cyclic homology of an algebra extension $\mathcal{B} \rightarrow \mathcal{A}$ is computed by the cyclic object

$$
\mathrm{C}_{n}(\mathcal{A} \mid \mathcal{B}):=\left[\mathcal{A}^{\otimes_{\mathcal{B}} n+1}\right]_{\mathcal{B}}
$$

equipped, for all $n \geq 0$, with the morphisms

$$
\begin{aligned}
& d_{i}: \mathrm{C}_{n}(\mathcal{A} \mid \mathcal{B}) \longrightarrow \mathrm{C}_{n-1}(\mathcal{A} \mid \mathcal{B}), \quad 0 \leq j \leq n, \\
& d_{i}\left[a^{0} \otimes_{\mathcal{B}} a^{1} \otimes_{\mathcal{B}} \cdots \otimes_{\mathcal{B}} a^{n}\right]_{\mathcal{B}} \\
& = \begin{cases}{\left[a^{0} a^{1} \otimes_{\mathcal{B}} \cdots \otimes_{\mathcal{B}} a^{n}\right]_{\mathcal{B}},} & i=0, \\
{\left[a^{0} \otimes_{\mathcal{B}} a^{1} \otimes_{\mathcal{B}} \cdots \otimes_{\mathcal{B}} a^{i} a^{i+1} \otimes_{\mathcal{B}} \cdots \otimes_{\mathcal{B}} a^{n}\right]_{\mathcal{B}},} & 1 \leq i \leq n-1, \\
{\left[a^{n} a^{0} \otimes_{\mathcal{B}} a^{1} \otimes_{\mathcal{B}} \cdots \otimes_{\mathcal{B}} a^{n-1}\right]_{\mathcal{B}},} & i=n,\end{cases} \\
& s_{i}: \mathrm{C}_{n}(\mathcal{A} \mid \mathcal{B}) \longrightarrow \mathrm{C}_{n+1}(\mathcal{A} \mid \mathcal{B}), \quad 0 \leq j \leq n, \\
& s_{i}\left[a^{0} \otimes_{\mathcal{B}} \cdots \otimes_{\mathcal{B}} a^{n}\right]_{\mathcal{B}}=\left[a^{0} \otimes_{\mathcal{B}} a^{1} \otimes_{\mathcal{B}} \cdots \otimes_{\mathcal{B}} a^{i} \otimes_{\mathcal{B}} 1 \otimes_{\mathcal{B}} a^{i+1} \otimes_{\mathcal{B}} \cdots \otimes_{\mathcal{B}} a^{n}\right]_{\mathcal{B}}
\end{aligned}
$$

and

$$
t_{n}: \mathrm{C}_{n}(\mathcal{A} \mid \mathcal{B}) \longrightarrow \mathrm{C}_{n}(\mathcal{A} \mid \mathcal{B}), \quad t_{n}\left[a^{0} \otimes_{\mathcal{B}} \cdots \otimes_{\mathcal{B}} a^{n}\right]_{\mathcal{B}}=\left[a^{n} \otimes_{\mathcal{B}} a^{0} \otimes_{\mathcal{B}} \cdots \otimes_{\mathcal{B}} a^{n-1}\right]_{\mathcal{B}} .
$$

Hochschild homology of the complex (2.5) is called the Hochschild homology of the extension, and is denoted by $\mathrm{HH}_{\bullet}(\mathcal{A} \mid \mathcal{B})$. Similarly, cyclic (resp. periodic cyclic, negative cyclic) homology of the cyclic object (2.5) is called the relative cyclic (resp. Hochschild, periodic cyclic, negative cyclic) homology of the extension $\mathcal{B} \rightarrow \mathcal{A}$, and it is denoted by $\operatorname{HC}_{\bullet}(\mathcal{A} \mid \mathcal{B})\left(\operatorname{resp} . \operatorname{HH}_{\bullet}(\mathcal{A} \mid \mathcal{B})\right.$, $\left.\mathrm{HP}_{\bullet}(\mathcal{A} \mid \mathcal{B}), \mathrm{HN}_{\bullet}(\mathcal{A} \mid \mathcal{B})\right)$.

The natural mapping

$$
\mathrm{C}_{n}(\mathcal{A}) \longrightarrow \mathrm{C}_{n}(\mathcal{A} \mid \mathcal{B}), \quad a^{0} \otimes \cdots \otimes a^{n} \mapsto\left[a^{0} \otimes_{\mathcal{B}} \cdots \otimes_{\mathcal{B}} a^{n}\right]_{\mathcal{B}}, \quad n \geq 0
$$

induces a map $\mathrm{HH}_{\bullet}(\mathcal{A}) \longrightarrow \mathrm{HH}_{\bullet}(\mathcal{A} \mid \mathcal{B})$ of Hochschild homology groups, which further induces a mapping $\mathrm{HC}_{\bullet}(\mathcal{A}) \longrightarrow \mathrm{HC}_{\bullet}(\mathcal{A} \mid \mathcal{B})$ of cyclic homology groups. This is the computational motivation behind the relative cyclic homology [29], in which it is proved to be an isomorphism when $\mathcal{B} \subseteq \mathcal{A}$ is a separable subalgebra (semisimple in characteristic zero). The relative cyclic homology then appeared in [36], where the relative cyclic homology $\mathrm{HC}_{\bullet}(k G \mid k N)$ of group algebras, associated to normal subgroups, was computed, and is applied to extend Eckmann's result [19] on the Bass conjecture.

Dually, for any $\mathcal{C}$-bicomodule $\mathcal{M}$ one defines $\mathcal{M}^{\mathcal{C}}:=\left\{m \in \mathcal{M} \mid m_{\langle 0\rangle} \otimes m_{\langle 1\rangle}=m_{\langle 0\rangle} \otimes m_{\langle-1\rangle}\right\}$. Then the relative cyclic cohomology of a coalgebra coextension $\mathcal{D} \rightarrow \mathcal{C}$ is computed by the cocyclic object

$$
\mathrm{C}^{n}(\mathcal{D} \mid \mathcal{C}):=\left(\mathcal{D}^{\square_{\mathcal{C}} n+1}\right)^{\mathcal{C}}, \quad n \geq 0,
$$

with the structure maps

$$
\begin{aligned}
& \delta_{i}: \mathrm{C}^{n}(\mathcal{D} \mid \mathcal{C}) \longrightarrow \mathrm{C}^{n+1}(\mathcal{D} \mid \mathcal{C}), \quad 0 \leq j \leq n+1, \\
& \delta_{i}\left(d^{0} \otimes d^{1} \otimes \cdots \otimes d^{n}\right)= \begin{cases}d^{0}{ }_{(1)} \otimes d^{0}{ }_{(2)} \otimes d^{1} \otimes \cdots \otimes d^{n}, & i=0, \\
d^{0} \otimes \cdots \otimes d_{(1)} \otimes d^{i}{ }_{(2)} \otimes \cdots \otimes d^{n}, & 1 \leq i \leq n, \\
d^{0}{ }_{(2)} \otimes d^{1} \otimes \cdots \otimes d^{n} \otimes d^{0}{ }_{(1)}, & i=n+1,\end{cases} \\
& \sigma_{j}: \mathrm{C}^{n}(\mathcal{D} \mid \mathcal{C}) \longrightarrow \mathrm{C}^{n-1}(\mathcal{D} \mid \mathcal{C}), \quad 0 \leq j \leq n-1, \\
& \sigma_{j}\left(d^{0} \otimes d^{1} \otimes \cdots \otimes d^{n}\right)=d^{0} \otimes d^{1} \otimes \cdots \otimes d^{j} \varepsilon\left(d^{j+1}\right) \otimes \cdots \otimes d^{n}
\end{aligned}
$$


and

$$
\tau_{n}: \quad \mathrm{C}^{n}(\mathcal{D} \mid \mathcal{C}) \longrightarrow \mathrm{C}^{n}(\mathcal{D} \mid \mathcal{C}), \quad \tau_{n}\left(d^{0} \otimes d^{1} \otimes \cdots \otimes d^{n}\right)=d^{1} \otimes \cdots \otimes d^{n} \otimes d^{0} .
$$

Then the cyclic duality $[15,31]$ yields the cyclic module structure on the collection of vector spaces

$$
\mathrm{C}_{n}(\mathcal{D} \mid \mathcal{C}):=\left(\mathcal{D}^{\square_{\mathcal{C}} n+1}\right)^{\mathcal{C}}
$$

given by faces

$$
\begin{aligned}
& d_{i}: \quad \mathrm{C}_{n}(\mathcal{D} \mid \mathcal{C}) \longrightarrow \mathrm{C}_{n-1}(\mathcal{D} \mid \mathcal{C}), \quad 0 \leq i \leq n, \\
& d_{i}\left(d^{0} \otimes d^{1} \otimes \cdots \otimes d^{n}\right)=d^{0} \otimes d^{1} \otimes \cdots \otimes \varepsilon\left(d^{i}\right) d^{i+1} \otimes \cdots \otimes d^{n}, \quad 0 \leq i \leq n-1, \\
& d_{n}\left(d^{0} \otimes d^{1} \otimes \cdots \otimes d^{n}\right)=d^{0} \otimes d^{1} \otimes \cdots \otimes d^{n-1} \varepsilon\left(d^{n}\right),
\end{aligned}
$$

degeneracies

$$
\begin{aligned}
& s_{i}: \quad \mathrm{C}_{n}(\mathcal{D} \mid \mathcal{C}) \longrightarrow \mathrm{C}_{n+1}(\mathcal{D} \mid \mathcal{C}), \quad 0 \leq i \leq n, \\
& s_{i}\left(d^{0} \otimes d^{1} \otimes \cdots \otimes d^{n}\right)=d^{0} \otimes d^{1} \otimes \cdots \otimes \Delta\left(d^{i}\right) \otimes \cdots \otimes d^{n},
\end{aligned}
$$

and the cyclic operator

$$
t_{n}: \quad \mathrm{C}_{n}(\mathcal{D} \mid \mathcal{C}) \longrightarrow \mathrm{C}_{n}(\mathcal{D} \mid \mathcal{C}), \quad t_{n}\left(d^{0} \otimes d^{1} \otimes \cdots \otimes d^{n}\right)=d^{n} \otimes d^{0} \otimes \cdots \otimes d^{n-1}
$$

Hochschild homology of the complex (2.7) is called the Hochschild homology of the coextension, and is denoted by $\mathrm{HH}_{\bullet}(\mathcal{D} \mid \mathcal{C})$. Similarly, cyclic (resp. periodic cyclic, negative cyclic) homology of the cyclic object (2.6) is called the relative cyclic (resp. Hochschild, periodic cyclic, negative cyclic) homology of the coextension $\mathcal{D} \rightarrow \mathcal{C}$, and it is denoted by $\mathrm{HC}_{\bullet}(\mathcal{D} \mid \mathcal{C})\left(\operatorname{resp} . \mathrm{HH}_{\bullet}(\mathcal{D} \mid \mathcal{C})\right.$, $\left.\mathrm{HP}_{\bullet}(\mathcal{D} \mid \mathcal{C}), \mathrm{HN}_{\bullet}(\mathcal{D} \mid \mathcal{C})\right)$

\subsection{Hopf-cyclic homology of $\mathcal{H}$-module coalgebras and $\mathcal{H}$-comodule algebras}

In this subsection we recall the relative Hopf-cyclic homology with coefficients, for coalgebras, using the cyclic duality principle [15,31].

Let us first recall the definition of a left-right stable anti-Yetter-Drinfeld module over a Hopf algebra $\mathcal{H}$ from [25]. Let a linear space $\mathcal{M}$ be a left $\mathcal{H}$-module by $\ell: \mathcal{H} \otimes \mathcal{M} \longrightarrow \mathcal{M}$ given by $\ell(h \otimes m)=h \cdot m$, and a right $\mathcal{H}$-comodule via $\rho: \mathcal{M} \longrightarrow \mathcal{M} \otimes \mathcal{H}$ given by $\rho(m)=m_{\langle 0\rangle} \otimes m_{\langle 1\rangle}$. Then $\mathcal{M}$ is called a left-right anti-Yetter-Drinfeld module (AYD module) over $\mathcal{H}$ if the $\mathcal{H}$-action and $\mathcal{H}$-coaction are compatible as

$$
(h \cdot m)_{\langle 0\rangle} \otimes(h \cdot m)_{\langle 1\rangle}=h_{(2)} \cdot m_{\langle 0\rangle} \otimes h_{(3)} m_{\langle 1\rangle} S\left(h_{(1)}\right) .
$$

A left-right AYD module $\mathcal{M}$ is called stable (and then is abbreviated as SAYD module) if

$$
m_{\langle 1\rangle} \cdot m_{\langle 0\rangle}=m \text {. }
$$

On the other hand, a right-left SAYD module structure is defined in terms of a right $\mathcal{H}$-module structure via $r: \mathcal{M} \otimes \mathcal{H} \longrightarrow \mathcal{M}$ given by $r(m \otimes h)=m \cdot h$, and a left $\mathcal{H}$-comodule structure via $\lambda: \mathcal{M} \longrightarrow \mathcal{H} \otimes \mathcal{M}$ given by $\lambda(m)=m_{\langle-1\rangle} \otimes m_{\langle 0\rangle}$ such that

$$
(m \cdot h)_{\langle-1\rangle} \otimes(m \cdot h)_{\langle 0\rangle}=S\left(h_{(3)}\right) \cdot m_{\langle-1\rangle} h_{(1)} \otimes m_{\langle 0\rangle} h_{(2)}, \quad m_{\langle 0\rangle} \cdot m_{\langle-1\rangle}=m .
$$


Note that these conditions are Pontryagin dual to each other. Indeed, the left-right SAYD module compatibility reads as commutativity of the following diagrams in the category $V$ ect of vector spaces ( $\sigma$ being the transposition of the corresponding tensorands)

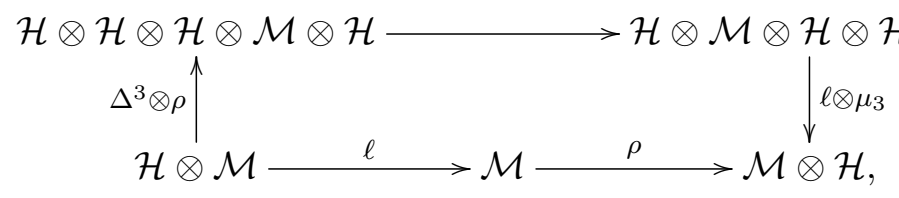

where the upper horizontal arrow admits two decompositions

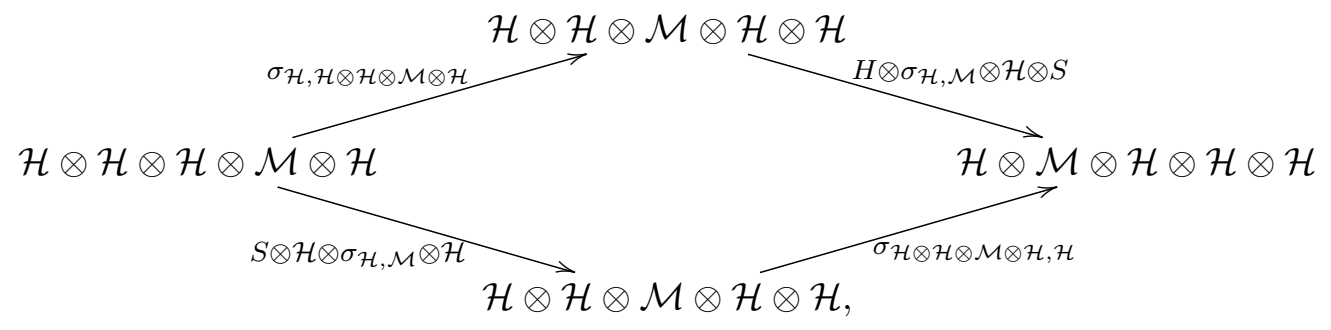

and

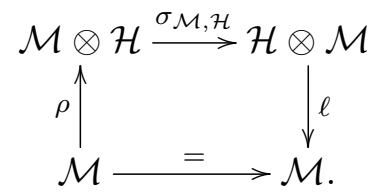

Now we see that reversing the arrows, inverting transpositions $\sigma$ and next interchanging in pairs $\Delta$ and $\mu, \ell$ and $\lambda, \rho$ and $r$, and finally left and right, we obtain the right-left SAYD module compatibility.

Let us now recall the Hopf-cyclic cohomology of a Hopf-module coalgebra with coefficients. Let $\mathcal{H}$ be a Hopf algebra with an invertible antipode, $\mathcal{C}$ a right $\mathcal{H}$-module coalgebra, i.e., a right $\mathcal{H}$-module such that

$$
\Delta(c \cdot h)=c_{(1)} \cdot h_{(1)} \otimes c_{(2)} \cdot h_{(2)}, \quad \varepsilon(c \cdot h)=\varepsilon(c) \varepsilon(h), \quad \forall c \in \mathcal{C}, \quad \forall h \in \mathcal{H},
$$

and $\mathcal{M}$ a left-right SAYD module over $\mathcal{H}$. Then for all $n \geq 0$ we define

$$
\mathrm{C}^{n}(\mathcal{C}, \mathcal{M})_{\mathcal{H}}:=\mathcal{C}^{\otimes n+1} \otimes_{\mathcal{H}} \mathcal{M},
$$

where on the right hand side the diagonal right $\mathcal{H}$-module structure on $\mathcal{C}^{\otimes n+1}$ is used.

The subscript $\mathcal{H}$ refers to the coinvariants of the diagonal right $\mathcal{H}$-module structure $\mathcal{C}^{\otimes n+1} \otimes \mathcal{M}$, by switching the one on $\mathcal{M}$ from left to right via the antipode. The collection of vector spaces $\mathrm{C}^{n}(\mathcal{C}, \mathcal{M})_{\mathcal{H}}$ together with the operators

$$
\begin{aligned}
& \delta_{i}: \quad \mathrm{C}^{n-1}(\mathcal{C}, \mathcal{M})_{\mathcal{H}} \rightarrow \mathrm{C}^{n}(\mathcal{C}, \mathcal{M})_{\mathcal{H}}, \quad 0 \leq i \leq n, \\
& \delta_{i}\left(\left(c^{0} \otimes \cdots \otimes c^{n-1}\right) \otimes_{\mathcal{H}} m\right) \\
& \quad \quad=\left(c^{0} \otimes \cdots \otimes c^{i}{ }_{(1)} \otimes c^{i}{ }_{(2)} \otimes \cdots \otimes c^{n-1}\right) \otimes_{\mathcal{H}} m, \quad 0 \leq i \leq n-1, \\
& \delta_{n}\left(\left(c^{0} \otimes \cdots \otimes c^{n-1}\right) \otimes_{\mathcal{H}} m\right)=\left(c^{0}{ }_{(2)} \otimes \cdots \otimes c^{n-1} \otimes c^{0}{ }_{(1)} \cdot S^{-1}\left(m_{\langle 1\rangle}\right)\right) \otimes \otimes_{\mathcal{H}} m_{\langle 0\rangle}, \\
& \sigma_{j}: \quad \mathrm{C}^{n+1}(\mathcal{C}, \mathcal{M})_{\mathcal{H}} \rightarrow \mathrm{C}^{n}(\mathcal{C}, \mathcal{M})_{\mathcal{H}}, \quad 0 \leq i \leq n, \\
& \sigma_{j}\left(\left(c^{0} \otimes \cdots \otimes c^{n+1}\right) \otimes_{\mathcal{H}} m\right)=\left(c^{0} \otimes \cdots \otimes c^{j} \varepsilon\left(c^{j+1}\right) \otimes \cdots \otimes c^{n+1}\right) \otimes_{\mathcal{H}} m,
\end{aligned}
$$

and

$$
\tau_{n}: \mathrm{C}^{n}(\mathcal{C}, \mathcal{M})_{\mathcal{H}} \rightarrow \mathrm{C}^{n}(\mathcal{C}, \mathcal{M})_{\mathcal{H}},
$$




$$
\tau_{n}\left(\left(c^{0} \otimes \cdots \otimes c^{n}\right) \otimes_{\mathcal{H}} m\right)=\left(c^{1} \otimes \cdots \otimes c^{n} \otimes c^{0} \cdot S^{-1}\left(m_{\langle 1\rangle}\right)\right) \otimes_{\mathcal{H}} m_{\langle 0\rangle} .
$$

is a cocyclic module. Cyclic cohomology of this cocyclic module is called the Hopf-cyclic cohomology of the $\mathcal{H}$-module coalgebra $\mathcal{C}$ with coefficients in the left-right SAYD module $\mathcal{M}$ over $\mathcal{H}$, and is denoted by $\mathrm{HC}^{\bullet}(\mathcal{C}, \mathcal{M})_{\mathcal{H}}$.

Applying the cyclic duality procedure $[15,31]$ we obtain on $(2.8)$ the cyclic module structure given by the faces

$$
\begin{aligned}
& d_{i}: \quad \mathrm{C}_{n+1}(\mathcal{C}, \mathcal{M})_{\mathcal{H}} \rightarrow \mathrm{C}_{n}(\mathcal{C}, \mathcal{M})_{\mathcal{H}}, \quad 0 \leq i \leq n+1, \\
& d_{i}\left(\left(c^{0} \otimes \cdots \otimes c^{n+1}\right) \otimes_{\mathcal{H}} m\right)=\left(c^{0} \otimes \cdots \otimes \varepsilon\left(c^{i}\right) c^{i+1} \otimes \cdots \otimes c^{n+1}\right) \otimes_{\mathcal{H}} m, \quad 0 \leq i \leq n, \\
& d_{n+1}\left(\left(c^{0} \otimes \cdots \otimes c^{n+1}\right) \otimes_{\mathcal{H}} m\right)=\left(\varepsilon\left(c^{n+1}\right) c^{0} \otimes \cdots \otimes c^{n}\right) \otimes_{\mathcal{H}} m
\end{aligned}
$$

degeneracies

$$
\begin{aligned}
& s_{i}: \mathrm{C}_{n-1}(\mathcal{C}, \mathcal{M})_{\mathcal{H}} \rightarrow \mathrm{C}_{n}(\mathcal{C}, \mathcal{M})_{\mathcal{H}}, \quad 0 \leq i \leq n-1, \\
& s_{i}\left(\left(c^{0} \otimes \cdots \otimes c^{n-1}\right) \otimes_{\mathcal{H}} m\right)=\left(c^{0} \otimes \cdots \otimes c^{i}{ }_{(1)} \otimes c^{i}{ }_{(2)} \otimes \cdots \otimes c^{n-1}\right) \otimes_{\mathcal{H}} m,
\end{aligned}
$$

and the cyclic operator

$$
\begin{aligned}
& t_{n}: \mathrm{C}_{n}(\mathcal{C}, \mathcal{M})_{\mathcal{H}} \rightarrow \mathrm{C}_{n}(\mathcal{C}, \mathcal{M})_{\mathcal{H}}, \\
& t_{n}\left(\left(c^{0} \otimes \cdots \otimes c^{n}\right) \otimes_{\mathcal{H}} m\right)=\left(c^{n} \cdot m_{\langle 1\rangle} \otimes c^{0} \otimes \cdots \otimes c^{n-1}\right) \otimes_{\mathcal{H}} m_{\langle 0\rangle} .
\end{aligned}
$$

The cyclic homology of this cyclic module is called the cyclic (resp. Hochschild, periodic cyclic, negative cyclic) homology of the $\mathcal{H}$-module coalgebra $\mathcal{C}$, with coefficients in the left-right SAYD module $\mathcal{M}$ over $\mathcal{H}$, and is denoted by $\mathrm{HC}_{\bullet}(\mathcal{C}, \mathcal{M})_{\mathcal{H}}\left(\operatorname{resp} . \mathrm{HH}_{\bullet}(\mathcal{C}, \mathcal{M})_{\mathcal{H}}, \operatorname{HP} \bullet(\mathcal{C}, \mathcal{M})_{\mathcal{H}}\right.$, $\left.\mathrm{HN}_{\bullet}(\mathcal{C}, \mathcal{M})_{\mathcal{H}}\right)$.

We conclude this section with the Hopf-cyclic homology of $\mathcal{H}$-comodule algebras [24]. Let $\mathcal{M}$ be a left-right SAYD module over the Hopf algebra $\mathcal{H}$, and $\mathcal{B}$ a left $\mathcal{H}$-comodule algebra. Then for $n \geq 0$ we define $\mathrm{C}_{n}(\mathcal{B}, \mathcal{M})^{\mathcal{H}}:=\mathcal{M} \square_{\mathcal{H}} \mathcal{B}^{\otimes n+1}$, where on the right hand side the diagonal left $\mathcal{H}$-comodule structure on $\mathcal{B}^{\otimes n+1}$ is used. The superscript $\mathcal{H}$ refers to the invariants of the diagonal right $\mathcal{H}$-comodule structure $\mathcal{M} \otimes \mathcal{B}^{\otimes n+1}$, switching the one on $\mathcal{B}^{\otimes n+1}$ from left to right by means of the antipode.

The collection of these vector spaces together with operators

$$
\begin{aligned}
& d_{i}: \mathrm{C}_{n}(\mathcal{B}, \mathcal{M})^{\mathcal{H}} \longrightarrow \mathrm{C}_{n-1}(\mathcal{B}, \mathcal{M})^{\mathcal{H}}, \quad 0 \leq i \leq n, \\
& d_{i}\left(m \otimes b^{0} \otimes \cdots \otimes b^{n}\right)=m \otimes b^{0} \otimes \cdots \otimes b^{i} b^{i+1} \otimes \cdots \otimes b^{n}, \quad 0 \leq i \leq n-1, \\
& d_{n}\left(m \otimes b^{0} \otimes \cdots \otimes b^{n}\right)=b^{n}{ }_{\langle-1\rangle} \cdot m \otimes b^{n}{ }_{\langle 0\rangle} b^{0} \otimes b^{1} \otimes \cdots \otimes b^{n-1}, \\
& s_{j}: \mathrm{C}_{n}(\mathcal{B}, \mathcal{M})^{\mathcal{H}} \longrightarrow \mathrm{C}_{n+1}(\mathcal{B}, \mathcal{M})^{\mathcal{H}}, \quad 0 \leq i \leq n, \\
& s_{j}\left(m \otimes b^{0} \otimes \cdots \otimes b^{n}\right)=m \otimes b^{0} \otimes \cdots \otimes b^{j} \otimes 1 \otimes \cdots \otimes b^{n},
\end{aligned}
$$

and

$$
\begin{aligned}
& t_{n}: \mathrm{C}_{n}(\mathcal{B}, \mathcal{M})^{\mathcal{H}} \longrightarrow \mathrm{C}_{n}(\mathcal{B}, \mathcal{M})^{\mathcal{H}} \\
& t_{n}\left(m \otimes b^{0} \otimes \cdots \otimes b^{n}\right)=b^{n}{ }_{\langle-1\rangle} \cdot m \otimes b^{n}{ }_{\langle 0\rangle} \otimes b^{0} \otimes \cdots \otimes b^{n-1}
\end{aligned}
$$

is a cyclic module. Finally, the cyclic homology of this cyclic module is called the cyclic (resp. Hochschild, periodic cyclic, negative cyclic) homology of the $\mathcal{H}$-comodule algebra $\mathcal{C}$, with coefficients in the left-right SAYD module $\mathcal{M}$ over $\mathcal{H}$, and is denoted by $\operatorname{HC} \bullet(\mathcal{C}, \mathcal{M})^{\mathcal{H}}$ (resp. $\left.\mathrm{HH}_{\bullet}(\mathcal{C}, \mathcal{M})^{\mathcal{H}}, \mathrm{HP}_{\bullet}(\mathcal{C}, \mathcal{M})^{\mathcal{H}}, \mathrm{HN}_{\bullet}(\mathcal{C}, \mathcal{M})^{\mathcal{H}}\right)$. 


\section{Relative cyclic homology as Hopf-cyclic homology with coefficients}

In this section we achieve our main result identifying the relative cyclic homology of a homogeneous $\mathcal{C}$-Galois extension $\mathcal{B} \subseteq \mathcal{H}$ with the Hopf-cyclic homology, with coefficients, of the right $\mathcal{H}$-module coalgebra $\mathcal{C}$. Moreover, in view of the Pontryagin duality of Subsection 2.2, we obtain an identification of the relative cyclic homology of a $\mathcal{B}$-Galois coextension $\mathcal{H} \rightarrow \mathcal{C}$ with the Hopf-cyclic homology, with (the Pontryagin dual) coefficients, of the $\mathcal{H}$-comodule algebra $\mathcal{B}$. We shall conclude the section developing spectral sequences to shed further light on the relative homology groups.

\subsection{The isomorphism of cyclic objects}

In this subsection we will construct an explicit isomorphism from the relative homology complex of a homogeneous $\mathcal{H} / \mathcal{I}$-Galois extension $\mathcal{B}:=\mathcal{H}^{\text {co } \mathcal{H} / \mathcal{I}} \rightarrow \mathcal{H}$ to the Hopf-cyclic homology complex of the $\mathcal{H}$-module coalgebra $\mathcal{H} / \mathcal{I}$.

Let $\mathcal{H}$ be a Hopf algebra and $\mathcal{I} \subseteq \mathcal{H}$ a coideal right ideal of $\mathcal{H}$. Then $\mathcal{H} / \mathcal{I}$ becomes a coaugmented quotient coalgebra in a canonical way,

$$
\Delta(\bar{h}):=\overline{h_{(1)}} \otimes \overline{h_{(2)}}, \quad \varepsilon(\bar{h}):=\varepsilon(h), \quad \forall h \in \mathcal{H},
$$

where $\bar{h}:=h+\mathcal{I}$, and the canonical coaugmentation of $\mathcal{H} / \mathcal{I}$ is given by the group-like $\overline{1}$.

Let $\mathcal{B} \subseteq \mathcal{H}$ be a homogeneous $\mathcal{H} / \mathcal{I}$-Galois extension given by the canonical right $\mathcal{H} / \mathcal{I}$-coaction

$$
\mathcal{H} \stackrel{\Delta}{\longrightarrow} \mathcal{H} \otimes \mathcal{H} \longrightarrow \mathcal{H} \otimes(\mathcal{H} / \mathcal{I}), \quad h \mapsto h_{(1)} \otimes \overline{h_{(2)}}
$$

on $\mathcal{H}$. In view of the definition $\mathcal{B}:=\mathcal{H}^{\text {co } \mathcal{H} / \mathcal{I}}$, which reads as

$$
b_{(1)} \otimes \overline{b_{(2)}}=b \otimes \overline{1},
$$

applying the counit to the left tensorands we deduce $\bar{b}=\varepsilon(b) \overline{1}$. We will use also the following iterated form of (3.1):

$$
b_{(1)} \otimes \cdots \otimes \overline{b_{(n+2)}}=b_{(1)} \otimes \cdots \otimes b_{(n+1)} \otimes \overline{1} .
$$

The three following facts are crucial to our purposes. First of all, $\mathcal{B}$ is the left coideal of $\mathcal{H}[11]$, i.e.,

$$
\Delta(\mathcal{B}) \subset \mathcal{H} \otimes \mathcal{B}
$$

Secondly, the Galois condition fixes the coideal right ideal $\mathcal{I} \subseteq \mathcal{H}$ completely as follows [11, Theorem 2.6].

Theorem 3.1. Let $\mathcal{B} \subseteq \mathcal{H}$ be a homogeneous $\mathcal{H} / \mathcal{I}$-extension. Then this extension is Galois if and only if $\mathcal{I}=\mathcal{B}^{+} \mathcal{H}$, where $\mathcal{B}^{+}:=\mathcal{B} \cap \operatorname{Ker} \varepsilon$.

Finally, there is an explicit formula for the translation map, and hence for the inverse to the canonical map, [11, Corollary 2.8].

Corollary 3.2. Let $\mathcal{B} \subseteq \mathcal{H}$ be a coalgebra-Galois $\mathcal{H} / \mathcal{I}$-extension as above. Then the translation map $\tau:=\operatorname{can}^{-1}(1 \otimes-)$ is given by

$$
\tau(\bar{h})=S\left(h_{(1)}\right) \otimes_{B} h_{(2)} .
$$


Moreover, it implies that the right hand side of (3.3) is independent of the choice of the representative $h$ of the class $\bar{h}$.

The canonical map

$$
\mathcal{H} \otimes_{\mathcal{B}} \mathcal{H} \rightarrow \mathcal{H} \otimes \mathcal{H} / \mathcal{I}, \quad h \otimes_{\mathcal{B}} h^{\prime} \mapsto h h_{(1)}^{\prime} \otimes \overline{h_{(2)}^{\prime}}
$$

and its inverse

$$
\mathcal{H} \otimes \mathcal{H} / \mathcal{I} \rightarrow \mathcal{H} \otimes_{\mathcal{B}} \mathcal{H}, \quad h \otimes \overline{h^{\prime}} \mapsto h S\left(h^{\prime}{ }_{(1)}\right) \otimes_{\mathcal{B}} h^{\prime}{ }_{(2)}
$$

can be inductively extended to $\mathcal{H}$-bimodule isomorphisms

$$
\begin{aligned}
& \operatorname{can}_{n}: \mathcal{M} \otimes_{\mathcal{B}} \otimes \mathcal{H}^{\otimes_{\mathcal{B}} n} \longrightarrow \mathcal{M} \otimes \mathcal{C}^{\otimes n}, \\
& \operatorname{can}_{n}\left(m \otimes_{\mathcal{B}} h^{1} \otimes_{\mathcal{B}} \cdots \otimes_{\mathcal{B}} h^{n}\right) \\
& =m h_{(1)}^{1} \ldots h_{(1)}^{n} \otimes \overline{h^{1}(2) \cdots h_{(2)}^{n_{(2)}}} \otimes \cdots \otimes \overline{h^{n-1}{ }_{(n)} h_{(n)}^{n_{(n)}}} \otimes \overline{h^{n}(n+1)} .
\end{aligned}
$$

and

$$
\begin{aligned}
& \operatorname{can}_{n}^{-1}: \mathcal{M} \otimes \mathcal{C}^{\otimes n} \longrightarrow \mathcal{M} \otimes_{\mathcal{B}} \mathcal{H}^{\otimes_{\mathcal{B}} n} \\
& \operatorname{can}_{n}^{-1}\left(m \otimes \overline{g^{1}} \otimes \cdots \otimes \overline{g^{n}}\right) \\
& \quad=m S\left(g_{(1)}^{1}\right) \otimes_{\mathcal{B}} g_{(2)}^{1} S\left(g_{(1)}^{2}\right) \otimes_{\mathcal{B}} \cdots \otimes_{\mathcal{B}} g^{n-1}{ }_{(2)} S\left(g^{n}{ }_{(1)}\right) \otimes_{\mathcal{B}} g^{n}{ }_{(2)},
\end{aligned}
$$

respectively, for any $\mathcal{H}$-bimodule $\mathcal{M}$, see also [30, Proposition 3.6].

We also recall that $\mathcal{H}$, equipped with the (left) adjoint $\mathcal{H}$-action

$$
h \triangleright h^{\prime}:=h_{(2)} h^{\prime} S\left(h_{(1)}\right)
$$

and the (right) $\mathcal{H}$-coaction given by the comultiplication, is a left-right SAYD module over $\mathcal{H}$, which is denoted by $\operatorname{ad}(\mathcal{H})$, see for instance [27, Example 4.3]. Moreover, this action satisfies

$$
h_{(2)} \triangleright\left(h^{\prime} h_{(1)}\right)=h_{(2)(2)} h^{\prime} h_{(1)} S\left(h_{(2)(1)}\right)=h_{(3)} h^{\prime} h_{(1)} S\left(h_{(2)}\right)=h_{(2)} h^{\prime} \varepsilon\left(h_{(1)}\right)=h h^{\prime} .
$$

All that is used in the following lemma.

Lemma 3.3. Let $\mathcal{B} \subseteq \mathcal{H}$ be a homogeneous $\mathcal{H} / \mathcal{I}$-Galois extension. Then for any $n \geq 0$ we have the isomorphism of vector spaces $\left[\mathcal{H}^{\otimes_{\mathcal{B}} n+1}\right]_{\mathcal{B}} \cong(\mathcal{H} / \mathcal{I})^{\otimes n+1} \otimes_{\mathcal{H}} \operatorname{ad}(\mathcal{H})$ implemented by

$$
\begin{aligned}
& \psi_{n}:(\mathcal{H} / \mathcal{I})^{\otimes n+1} \otimes_{\mathcal{H}} \operatorname{ad}(\mathcal{H}) \stackrel{\cong}{\longrightarrow}\left[\mathcal{H}^{\otimes{ }_{\mathcal{B}} n+1}\right]_{\mathcal{B}}, \\
& \psi_{n}\left(\left(\overline{g^{0}} \otimes \cdots \otimes \overline{g^{n}}\right) \otimes_{\mathcal{H}} h\right)=\left[g^{n}{ }_{(2)} h S\left(g^{0}{ }_{(1)}\right) \otimes_{\mathcal{B}} g^{0}{ }_{(2)} S\left(g^{1}{ }_{(1)}\right) \otimes_{\mathcal{B}} \cdots \otimes_{\mathcal{B}} g^{n-1}{ }_{(2)} S\left(g^{n}{ }_{(1)}\right)\right]_{\mathcal{B}},
\end{aligned}
$$

with the inverse

$$
\begin{aligned}
& \varphi_{n}:\left[\mathcal{H}^{\otimes_{\mathcal{B}} n+1}\right]_{\mathcal{B}} \cong(\mathcal{H} / \mathcal{I})^{\otimes n+1} \otimes_{\mathcal{H}} \operatorname{ad}(\mathcal{H}), \\
& \varphi_{n}\left(\left[h^{0} \otimes_{\mathcal{B}} \cdots \otimes_{\mathcal{B}} h^{n}\right]_{\mathcal{B}}\right) \\
& \quad=\left(\overline{h^{1}{ }_{(2)} \cdots h^{n}(2)} \otimes \cdots \otimes \overline{h^{n-1}{ }_{(n)} h^{n_{(n)}}} \otimes \overline{h^{n}(n+1)} \otimes \overline{1}\right) \otimes_{\mathcal{H}} h^{0} h^{1}{ }_{(1)} \cdots h^{n}{ }_{(1)} .
\end{aligned}
$$

Proof. First of all, we have to prove that the maps are well defined. Let us begin with (3.7). We observe that

$$
\begin{aligned}
\varphi_{n}( & {\left.\left[h^{0} \otimes_{\mathcal{B}} \cdots \otimes_{\mathcal{B}} h^{n} b\right]_{\mathcal{B}}\right) } \\
& =\left(\overline{h^{1}(2) \cdots\left(h^{n} b\right)_{(2)}} \otimes \cdots \otimes \overline{h^{n-1}\left(_{(n)}\left(h^{n} b\right)_{(n)}\right.} \otimes \overline{\left(h^{n} b\right)_{(n+1)}} \otimes \overline{1}\right) \otimes_{\mathcal{H}} h^{0} h_{(1)}^{1} \cdots\left(h^{n} b\right)_{(1)}
\end{aligned}
$$




$$
\begin{aligned}
& =\left(\overline{h^{1_{(2)}} \cdots h_{(2)}^{n_{(2)}} b_{(2)}} \otimes \cdots \otimes \overline{h^{n-1}{ }_{(n)} h_{(n)}^{n_{(n)}} b_{(n)}} \otimes \overline{h_{(n+1)}^{n_{(n+1)}}} \otimes \overline{1}\right) \otimes_{\mathcal{H}} h^{0} h_{(1)}^{1} \cdots h_{(1)}^{n} b_{(1)} \\
& =\left(\overline{h^{1}(2)} \cdots h^{n_{(2)}} b_{(2)} \otimes \cdots \otimes \overline{h^{n-1}{ }_{(n)} h_{(n)}^{n_{(n)}}} b_{(n)} \otimes \overline{h^{n_{(n+1)}}} b_{(n+1)} \otimes \overline{1}\right) \otimes_{\mathcal{H}} h^{0} h_{(1)}^{1} \cdots h_{(1)}^{n} b_{(1)} \\
& \stackrel{(3.2)}{=}\left(\overline{h^{1}(2)} \cdots h_{(2)}^{n} b_{(2)} \otimes \cdots \otimes \overline{h^{n-1}{ }_{(n)} h_{(n)}^{n_{(n)}}} b_{(n)} \otimes \overline{h_{(n+1)}^{n}} b_{(n+1)} \otimes \overline{b_{(n+2)}}\right) \\
& \otimes_{\mathcal{H}} h^{0} h_{(1)}^{1} \cdots h_{(1)}^{n} b_{(1)} \\
& =\left(\overline{h_{(2)}^{1} \cdots h^{n}(2)} b_{(2)} \otimes \cdots \otimes \overline{h^{n-1}{ }_{(n)} h_{(n)}^{n_{(n)}}} b_{(n)} \otimes \overline{h^{n_{(n+1)}}} b_{(n+1)} \otimes \overline{1} b_{(n+2)}\right) \\
& \otimes_{\mathcal{H}} h^{0} h_{(1)}^{1} \cdots h_{(1)}^{n} b_{(1)} \\
& =\left(\overline{h^{1}{ }_{(2)} \cdots h_{(2)}^{n_{(2)}}} \otimes \cdots \otimes \overline{h^{n-1}\left(_{(n)} h^{n}(n)\right.} \otimes \overline{h_{(n+1)}^{n_{(n+1)}}} \otimes \overline{1}\right) b_{(2)} \otimes_{\mathcal{H}} h^{0} h_{(1)}^{1} \cdots h_{(1)}^{n} b_{(1)} \\
& =\left(\overline{h^{1}{ }_{(2)} \cdots h^{n}(2)} \otimes \cdots \otimes \overline{h^{n-1}{ }_{(n)} h^{n}(n)} \otimes \overline{h^{n}(n+1)} \otimes \overline{1}\right) \otimes_{\mathcal{H}} b_{(2)} \triangleright\left(h^{0} h_{(1)}^{1} \cdots h_{(1)}^{n_{(1)}} b_{(1)}\right) \\
& \stackrel{(3.5)}{=}\left(\overline{h^{1}{ }_{(2)} \cdots h_{(2)}^{n}} \otimes \cdots \otimes \overline{h^{n-1}{ }_{(n)} h^{n}{ }_{(n)}} \otimes \overline{h^{n}(n+1)} \otimes \overline{1}\right) \otimes_{\mathcal{H}} b h^{0} h_{(1)}^{1} \cdots h_{(1)}^{n} \\
& =\varphi_{n}\left(\left[b h^{0} \otimes_{\mathcal{B}} \cdots \otimes_{\mathcal{B}} h^{n}\right]_{\mathcal{B}}\right) \text {, }
\end{aligned}
$$

that

$$
\begin{aligned}
& \varphi_{n}\left(\left[h^{0} \otimes_{\mathcal{B}} b h^{1} \otimes_{\mathcal{B}} \cdots \otimes_{\mathcal{B}} h^{n}\right]_{\mathcal{B}}\right) \\
& =\left(\overline{\left(b h^{1}\right)_{(2)} \cdots h_{(2)}^{n}} \otimes \cdots \otimes \overline{h^{n-1}{ }_{(n)} h_{(n)}^{n}} \otimes \overline{h_{(n+1)}^{n}} \otimes \overline{1}\right) \otimes_{\mathcal{H}} h^{0}\left(b h^{1}\right)_{(1)} \cdots h_{(1)}^{n} \\
& =\left(\overline{b_{(2)} h_{(2)}^{1} \cdots h_{(2)}^{n_{2}}} \otimes \cdots \otimes \overline{h^{n-1_{(n)}} h_{(n)}^{n_{(n)}}} \otimes \overline{h^{n_{(n+1)}}} \otimes \overline{1}\right) \otimes_{\mathcal{H}} h^{0} b_{(1)} h_{(1)}^{1_{1}} \cdots h_{(1)}^{n} \\
& =\left(\overline{b_{(2)}} h_{(2)}^{1} \cdots h_{(2)}^{n} \otimes \cdots \otimes \overline{h^{n-1}{ }_{(n)} h_{(n)}^{n_{(n)}}} \otimes \overline{h^{n_{(n+1)}}} \otimes \overline{1}\right) \otimes_{\mathcal{H}} h^{0} b_{(1)} h_{(1)}^{1} \cdots h_{(1)}^{n} \\
& \stackrel{(3.1)}{=}\left(\overline{1} h_{(2)}^{1} \cdots h_{(2)}^{n} \otimes \cdots \otimes \overline{h^{n-1}{ }_{(n)} h_{(n)}^{n}} \otimes \overline{h^{n}(n+1)} \otimes \overline{1}\right) \otimes \mathcal{H}^{0} b h_{(1)}^{1} \cdots h_{(1)}^{n} \\
& =\left(\overline{h^{1}(2) \cdots h^{n}{ }_{(2)}} \otimes \cdots \otimes \overline{h^{n-1}{ }_{(n)} h^{n}(n)} \otimes \overline{h^{n}(n+1)} \otimes \overline{1}\right) \otimes_{\mathcal{H}} h^{0} b h_{(1)}^{1} \cdots h_{(1)}^{n} \\
& =\varphi_{n}\left(\left[h^{0} b \otimes_{\mathcal{B}} h^{1} \otimes_{\mathcal{B}} \cdots \otimes_{\mathcal{B}} h^{n}\right]_{\mathcal{B}}\right) \text {, }
\end{aligned}
$$

and that

$$
\begin{aligned}
& \varphi_{n}\left(\left[h^{0} \otimes_{\mathcal{B}} \cdots \otimes_{\mathcal{B}} h^{i} \otimes_{\mathcal{B}} b h^{i+1} \otimes_{\mathcal{B}} \cdots \otimes_{\mathcal{B}} h^{n}\right]_{\mathcal{B}}\right) \\
& =\left(\overline{h^{1}{ }_{(2)} \cdots h^{i_{(2)}}\left(b h^{i+1}\right)_{(2)} \cdots h^{n}{ }_{(2)}} \otimes \cdots \otimes \overline{h^{i_{(i+1)}}\left(b h^{i+1}\right)_{(i+1)} \cdots h^{n_{(i+1)}}}\right. \\
& \left.\otimes \overline{\left(b h^{i+1}\right)_{(i+2)} \cdots h_{(i+2)}^{n}} \otimes \overline{h^{i+2}{ }_{(i+3)} \cdots h^{n}{ }_{(i+3)}} \otimes \cdots \otimes \overline{h^{n}(n+1)} \otimes \overline{1}\right) \\
& \otimes_{\mathcal{H}} h^{0} h^{1}{ }_{(1)} \cdots h_{(1)}^{i}\left(b h^{i+1}\right)_{(1)} \cdots h_{(1)}^{n} \\
& =\left(\overline{h^{1}{ }_{(2)} \cdots h_{(2)}^{i_{(2)}} b_{(2)} h^{i+1}{ }_{(2)} \cdots h^{n}(2)} \otimes \cdots \otimes \overline{h_{(i+1)}^{i_{(i+1)}} h^{i+1}{ }_{(i+1)} \cdots h^{n}(i+1)}\right. \\
& \left.\otimes \overline{b_{(i+2)} h^{i+1}{ }_{(i+2)} \cdots h_{(i+2)}^{n_{(i+2)}}} \otimes \overline{h^{i+2}{ }_{(i+3)} \cdots h^{n_{(i+3)}}} \otimes \cdots \otimes \overline{h^{n_{(n+1)}}} \otimes \overline{1}\right) \\
& \otimes_{\mathcal{H}} h^{0} h_{(1)}^{1} \cdots h_{(1)}^{i} b_{(1)} h^{i+1}{ }_{(1)} \cdots h_{(1)}^{n} \\
& =\left(\overline{h^{1}{ }_{(2)} \cdots h_{(2)}^{i} b_{(2)} h^{i+1}{ }_{(2)} \cdots h^{n}(2)} \otimes \cdots \otimes \overline{h_{(i+1)}^{i_{(i+1)}} h^{i+1}{ }_{(i+1)} \cdots h^{n}{ }_{(i+1)}}\right. \\
& \left.\otimes \overline{b_{(i+2)}} h^{i+1}{ }_{(i+2)} \cdots h_{(i+2)}^{n} \otimes \overline{h^{i+2}{ }_{(i+3)} \cdots h^{n}{ }_{(i+3)}} \otimes \cdots \otimes \overline{h^{n}(n+1)} \otimes \overline{1}\right) \\
& \otimes_{\mathcal{H}} h^{0} h_{(1)}^{1} \cdots h_{(1)}^{i} b_{(1)} h^{i+1}{ }_{(1)} \cdots h_{(1)}^{n} \\
& \stackrel{(3.2)}{=}\left(\overline{h^{1}{ }_{(2)} \cdots h_{(2)}^{i_{(2)}} b^{i+1_{(2)}} \cdots h^{n}{ }_{(2)}} \otimes \cdots \otimes \overline{h_{(i+1)}^{i_{(i+1)}} b^{i+1}{ }_{(i+1)} \cdots h^{n}{ }_{(i+1)}}\right.
\end{aligned}
$$

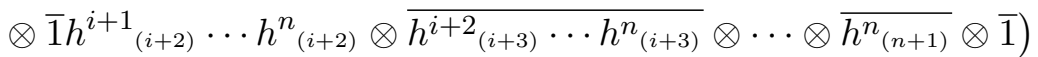

$$
\begin{aligned}
& \otimes_{\mathcal{H}} h^{0} h_{(1)}^{1} \cdots h_{(1)}^{i} b_{(1)} h^{i+1}{ }_{(1)} \cdots h_{(1)}^{n} \\
& =\left(\overline{h^{1}(2) \cdots\left(h^{i} b\right)_{(2)} h^{i+1}{ }_{(2)} \cdots h_{(2)}^{n}} \otimes \cdots \otimes \overline{\left(h^{i} b\right)_{(i+1)} h^{i+1}{ }_{(i+1)} \cdots h^{n}{ }_{(i+1)}}\right. \\
& \left.\otimes \overline{h^{i+1}{ }_{(i+2)} \cdots h^{n}{ }_{(i+2)}} \otimes \overline{h^{i+2}{ }_{(i+3)} \cdots h^{n_{(i+3)}}} \otimes \cdots \otimes \overline{h^{n}(n+1)} \otimes \overline{1}\right) \\
& \otimes_{\mathcal{H}} h^{0} h^{1}{ }_{(1)} \cdots\left(h^{i} b\right)_{(1)} h^{i+1}{ }_{(1)} \cdots h^{n}{ }_{(1)}
\end{aligned}
$$




$$
=\varphi_{n}\left(\left[h^{0} \otimes_{\mathcal{B}} \cdots \otimes_{\mathcal{B}} h^{i} b \otimes_{\mathcal{B}} h^{i+1} \otimes_{\mathcal{B}} \cdots \otimes_{\mathcal{B}} h^{n}\right]_{\mathcal{B}}\right) .
$$

As for (3.6), we first note that by (3.3) the right hand side depends only on the representatives appearing on the left hand side. Moreover, for any $p \in \mathcal{H}$,

$$
\begin{aligned}
\psi_{n}( & \left.\left(\overline{g^{0}} \otimes \cdots \otimes \overline{g^{n}}\right) \cdot p \otimes_{\mathcal{H}} h\right)=\psi_{n}\left(\left(\overline{g^{0} p_{(1)}} \otimes \cdots \otimes \overline{g^{n} p_{(n+1)}}\right) \otimes_{\mathcal{H}} h\right) \\
= & {\left[g^{n}{ }_{(2)} p_{(n+1)} h S\left(g^{0}{ }_{(1)} p_{(1)}\right) \otimes_{\mathcal{B}} g_{(2)}^{0} p_{(2)} S\left(g_{(1)}^{1} p_{(3)}\right) \otimes_{\mathcal{B}} \cdots\right.} \\
& \left.\otimes_{\mathcal{B}} g^{n-1_{(2)}} p_{(2 n)} S\left(g^{n}{ }_{(1)} p_{(2 n+1)}\right)\right]_{\mathcal{B}} \\
= & {\left[g^{n}{ }_{(2)} p_{(n+1)} h S\left(p_{(1)}\right) S\left(g_{(1)}^{0}\right) \otimes_{\mathcal{B}} g_{(2)}^{0} p_{(2)} S\left(p_{(3)}\right) S\left(g^{1}{ }_{(1)}\right) \otimes_{\mathcal{B}} \cdots\right.} \\
& \left.\otimes_{\mathcal{B}} g^{n-1}{ }_{(2)} p_{(2 n)} S\left(p_{(2 n+1)}\right) S\left(g^{n}{ }_{(1)}\right)\right]_{\mathcal{B}} \\
= & {\left[g^{n}{ }_{(2)} p_{(2)} h S\left(p_{(1)}\right) S\left(g^{0}{ }_{(1)}\right) \otimes_{\mathcal{B}} g^{0}{ }_{(2)} S\left(g_{(1)}^{1}\right) \otimes_{\mathcal{B}} \cdots \otimes_{\mathcal{B}} g^{n-1}{ }_{(2)} S\left(g^{n}{ }_{(1)}\right)\right]_{\mathcal{B}} } \\
= & \psi_{n}\left(\left(\overline{g^{0}} \otimes \cdots \otimes \overline{g^{n}}\right) \otimes_{\mathcal{H}} p \triangleright h\right) .
\end{aligned}
$$

Accordingly, $\psi_{n}$ is well-defined for any $n \geq 0$. have

Finally, we prove that $\varphi_{n}$ and $\psi_{n}$ are inverses to each other for any $n \geq 0$. On one hand we

$$
\begin{aligned}
& \left(\psi_{n} \circ \varphi_{n}\right)\left(\left[h^{0} \otimes_{\mathcal{B}} \cdots \otimes_{\mathcal{B}} h^{n}\right]_{\mathcal{B}}\right)
\end{aligned}
$$

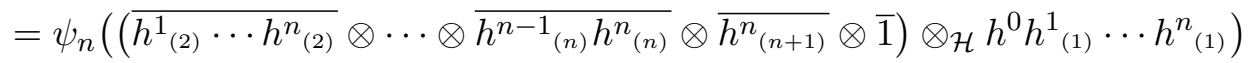

$$
\begin{aligned}
& =\left[1_{(2)} h^{0} h_{(1)}^{1} \cdots h_{(1)}^{n} S\left(\left(h_{(2)}^{1} \cdots h_{(2)}^{n}\right)_{(1)}\right) \otimes_{\mathcal{B}}\left(h_{(2)}^{1} \cdots h_{(2)}^{n}\right)_{(2)} S\left(\left(h_{(3)}^{2} \cdots h_{(3)}^{n}\right)_{(1)}\right)\right. \\
& \left.\otimes_{\mathcal{B}} \cdots \otimes_{\mathcal{B}}\left(h^{n-1_{(n)}} h_{(n)}^{n}\right)_{(2)} S\left(h_{(n+1)(1)}^{n}\right) \otimes_{\mathcal{B}} h_{(n+1)(2)}^{n} S\left(1_{(1)}\right)\right]_{\mathcal{B}}
\end{aligned}
$$

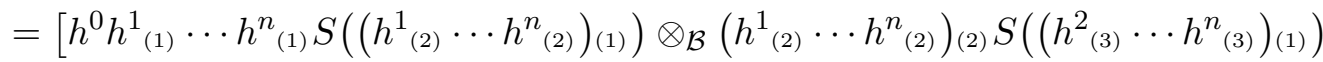

$$
\begin{aligned}
& \left.\otimes_{\mathcal{B}} \cdots \otimes_{\mathcal{B}}\left(h_{(n)}^{n-1} h_{(n)}^{n}\right)_{(2)} S\left(h_{(n+1)(1)}^{n}\right) \otimes_{\mathcal{B}} h_{(n+1)(2)}^{n}\right]_{\mathcal{B}}
\end{aligned}
$$

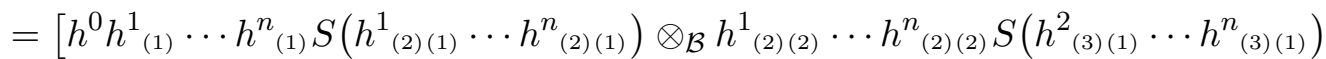

$$
\begin{aligned}
& \left.\otimes_{\mathcal{B}} \cdots \otimes_{\mathcal{B}} h_{(n)(2)}^{n-1} h_{(n)(2)}^{n} S\left(h_{(n+1)(1)}^{n}\right) \otimes_{\mathcal{B}} h_{(n+1)(2)}^{n}\right]_{\mathcal{B}} \\
& =\left[h^{0} \cdot h_{(1)}^{1_{(1)}} \cdots h_{(1)}^{n} S\left(h_{(2)}^{n}\right) \cdots S\left(h_{(2)}^{1}\right) \otimes_{\mathcal{B}} h_{(3)}^{1} \cdot h_{(3)}^{2} \cdots h_{(3)}^{n} S\left(h_{(3)}^{n}\right) \cdots S\left(h_{(3)}^{2}\right)\right. \\
& \left.\otimes_{\mathcal{B}} \cdots \otimes_{\mathcal{B}} h^{n-1}{ }_{(2 n-1)} h_{(2 n-1)}^{n} S\left(h_{(2 n)}^{n}\right) \otimes_{\mathcal{B}} h^{n}{ }_{(2 n+1)}\right]_{\mathcal{B}} \\
& =\left[h^{0} \cdot h_{(1)}^{1} \cdots h_{(1)}^{n} S\left(h_{(2)}^{n}\right) \cdots S\left(h_{(2)}^{1}\right) \otimes_{\mathcal{B}} h_{(3)}^{1} \cdot h_{(3)}^{2} \cdots h_{(3)}^{n} S\left(h_{(3)}^{n}\right) \cdots S\left(h_{(3)}^{2}\right)\right. \\
& \left.\otimes_{\mathcal{B}} \cdots \otimes_{\mathcal{B}} h^{n-1}{ }_{(2 n-1)} h_{(2 n-1)}^{n} S\left(h^{n}{ }_{(2 n)}\right) \otimes_{\mathcal{B}} h^{n}{ }_{(2 n+1)}\right]_{\mathcal{B}} \\
& =\left[h^{0} \cdot h_{(1)}^{1} \cdots h_{(1)}^{n-1} S\left(h_{(2)}^{n-1}\right) \cdots S\left(h_{(2)}^{1}\right)\right. \\
& \left.\otimes_{\mathcal{B}} h_{(3)}^{1} \cdot h_{(3)}^{2} \cdots h^{n-1}{ }_{(3)} S\left(h^{n-1}{ }_{(3)}\right) \cdots S\left(h_{(3)}^{2}\right) \otimes_{\mathcal{B}} \cdots \otimes_{\mathcal{B}} h^{n-1}{ }_{(2 n-1)} \otimes_{\mathcal{B}} h^{n}\right]_{\mathcal{B}}=\cdots \\
& =\left[h^{0} \otimes_{\mathcal{B}} \cdots \otimes_{\mathcal{B}} h^{n}\right]_{\mathcal{B}} \text {, }
\end{aligned}
$$

while on the other hand

$$
\begin{aligned}
& \left(\varphi_{n} \circ \psi_{n}\right)\left(\left(\overline{g^{0}} \otimes \cdots \otimes \overline{g^{n}}\right) \otimes_{\mathcal{H}} h\right) \\
& =\varphi_{n}\left(\left[g_{(2)}^{n} h S\left(g_{(1)}^{0}\right) \otimes_{\mathcal{B}} g_{(2)}^{0} S\left(g_{(1)}^{1}\right) \otimes_{\mathcal{B}} \cdots \otimes_{\mathcal{B}} g^{n-1}{ }_{(2)} S\left(g_{(1)}^{n}\right)\right]_{\mathcal{B}}\right)
\end{aligned}
$$

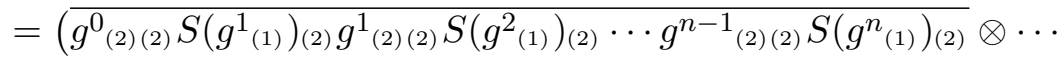

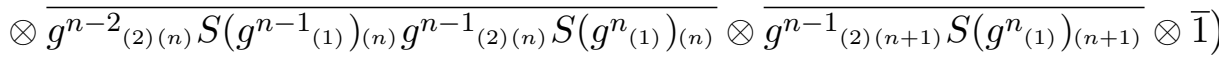

$$
\begin{aligned}
& \otimes_{\mathcal{H}} g_{(2)}^{n} h S\left(g_{(1)}^{0}\right) g_{(2)(1)}^{0} S\left(g_{(1)}^{1_{(1)}} g_{(2)(1)}^{1_{(1)}} S\left(g_{(1)}^{2}\right)_{(1)} \cdots g^{n-1_{(2)(1)}} S\left(g_{(1)}^{n}\right)_{(1)}\right.
\end{aligned}
$$

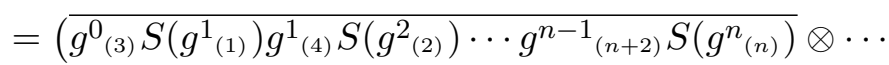

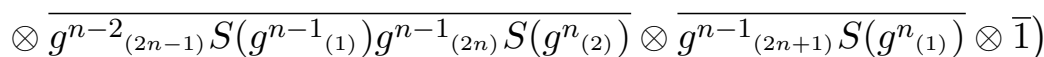

$$
\begin{aligned}
& \otimes_{\mathcal{H}} g_{(n+2)}^{n_{(n+2}} h S\left(g_{(1)}^{0}\right) g_{(2)}^{0} S\left(g_{(2)}^{1}\right) g_{(3)}^{1_{(3)}} S\left(g_{(3)}^{2}\right) \cdots g^{n-1}{ }_{(n+1)} S\left(g_{(n+1)}^{n}\right)
\end{aligned}
$$




$$
\begin{aligned}
& =\left(\overline{g^{0} S\left(g^{\left.n_{(n)}\right)}\right.} \otimes \cdots \otimes \overline{g^{n-2} S\left(g^{\left.n_{(2)}\right)}\right.} \otimes \overline{g^{n-1} S\left(g^{\left.n_{(1)}\right)}\right.} \otimes \overline{1}\right) \otimes_{\mathcal{H}} g_{(n+2)}^{n_{(n)}} h\left(g_{(n+1)}^{n_{(n)}}\right) \\
& =\left(\overline{g^{0} S\left(g^{\left.n_{(1)(n)}\right)}\right.} \otimes \cdots \otimes \overline{g^{n-1} S\left(g^{\left.n_{(1)(1)}\right)}\right.} \otimes \overline{1}\right) \otimes_{\mathcal{H}} g^{n}(2) h S\left(g^{n}{ }_{(1)(n+1)}\right) \\
& =\left(\left(\overline{g^{0}} \otimes \cdots \otimes \overline{g^{n-1}}\right) \cdot S\left(g_{(1)}^{n}\right) \otimes \overline{1}\right) \otimes_{\mathcal{H}} g_{(2)}^{n} \triangleright h \\
& =\left(\left(\overline{g^{0}} \otimes \cdots \otimes \overline{g^{n-1}}\right) \cdot S\left(g_{(1)}^{n}\right) \otimes \overline{1}\right) \cdot g_{(2)}^{n} \otimes_{\mathcal{H}} h \\
& =\left(\overline{g^{0}} \otimes \cdots \otimes \overline{g^{n-1}} \otimes \overline{g^{n}}\right) \otimes \mathcal{H} h .
\end{aligned}
$$

We are now ready to state our main result.

Theorem 3.4. Let $\mathcal{I} \subseteq \mathcal{H}$ be a coideal right ideal in a Hopf algebra $\mathcal{H}$ such that $\mathcal{H}^{\text {co } \mathcal{H} / \mathcal{I}} \subseteq \mathcal{H}$ is an $\mathcal{H} / \mathcal{I}$-Galois extension. Let also $\operatorname{ad}(\mathcal{H})=\mathcal{H}$ be the left-right $S A Y D$ module with the right adjoint action, and the left coaction given by the comultiplication of $\mathcal{H}$. Then there exists an isomorphism

$$
\psi_{n}: \mathrm{C}_{n}(\mathcal{H} / \mathcal{I}, \operatorname{ad}(\mathcal{H}))_{\mathcal{H}} \longrightarrow \mathrm{C}_{n}\left(\mathcal{H} \mid \mathcal{H}^{\operatorname{co} \mathcal{H} / \mathcal{I}}\right)
$$

of cyclic modules, defined by (3.6), (3.7).

Proof. Let us, as above, adopt the notation $\mathcal{B}:=\mathcal{H}^{\text {co } \mathcal{H} / \mathcal{I}}$. We shall first go through the commutation with the face operators. For $i=0$,

$$
\begin{aligned}
& \varphi_{n-1} d_{0}\left(\left[h^{0} \otimes_{\mathcal{B}} \cdots \otimes_{\mathcal{B}} h^{n}\right]_{\mathcal{B}}\right)=\varphi_{n-1}\left(\left[h^{0} h^{1} \otimes_{\mathcal{B}} \cdots \otimes_{\mathcal{B}} h^{n}\right]_{\mathcal{B}}\right) \\
& =\left(\overline{h^{2}{ }_{(2)} \cdots h^{n}{ }_{(2)}} \otimes \cdots \otimes \overline{h^{n-1}{ }_{(n-1)} h_{(n-1)}^{n}} \otimes \overline{h^{n}{ }_{(n)}} \otimes \overline{1}\right) \otimes \mathcal{H}^{0} h^{1} h_{(1)}^{2} \cdots h^{n}{ }_{(1)} \\
& =d_{0} \varphi_{n}\left(\left[h^{0} \otimes_{\mathcal{B}} \cdots \otimes_{\mathcal{B}} h^{n}\right]_{\mathcal{B}}\right) \text {. }
\end{aligned}
$$

For $1 \leq i \leq n-1$, we observe that

$$
\begin{aligned}
& \varphi_{n-1} d_{i}\left(\left[h^{0} \otimes_{\mathcal{B}} \cdots \otimes_{\mathcal{B}} h^{n}\right]_{\mathcal{B}}\right)=\varphi_{n-1}\left(\left[h^{0} \otimes_{\mathcal{B}} \cdots \otimes_{\mathcal{B}} h^{i} h^{i+1} \otimes_{\mathcal{B}} \cdots \otimes_{\mathcal{B}} h^{n}\right]_{\mathcal{B}}\right) \\
& =\left(\overline{h^{1}(2) \cdots\left(h^{i} h^{i+1}\right)_{(2)} \cdots h_{(2)}^{n}} \otimes \cdots \otimes \overline{\left(h^{i} h^{i+1}\right)_{(i+1)} \cdots h^{n}(i+1)}\right. \\
& \left.\otimes \overline{h^{i+2}{ }_{(i+2)} \cdots h^{n}{ }_{(i+2)}} \otimes \cdots \otimes \overline{h^{n-1}{ }_{(n-1)} h^{n}{ }_{(n-1)}} \otimes \overline{h^{n}(n)} \otimes \overline{1}\right) \\
& \otimes_{\mathcal{H}} h^{0} h_{(1)}^{1} \cdots\left(h^{i} h^{i+1}\right)_{(1)} \cdots h_{(1)}^{n} \\
& =\left(\overline{h^{1}{ }_{(2)} \cdots h^{n}{ }_{(2)}} \otimes \cdots \otimes \overline{h_{(i+1)}^{i} h^{i+1}{ }_{(i+1)} \cdots h^{n}{ }_{(i+1)}} \otimes \varepsilon\left(\overline{h^{i+1}{ }_{(i+2)} \cdots h^{n}{ }_{(i+2)}}\right) \otimes \cdots\right. \\
& \left.\otimes \overline{h^{n-1}(n) h_{(n)}^{n}} \otimes \overline{h^{n}(n+1)} \otimes \overline{1}\right) \otimes \mathcal{H} h^{0} h_{(1)}^{1} \cdots h_{(1)}^{n} \\
& =d_{i} \varphi_{n}\left(\left[h^{0} \otimes_{\mathcal{B}} \cdots \otimes_{\mathcal{B}} h^{n}\right]_{\mathcal{B}}\right) \text {. }
\end{aligned}
$$

Finally for the last face operator we have

$$
\begin{aligned}
& \varphi_{n-1} d_{n}\left(\left[h^{0} \otimes_{\mathcal{B}} \cdots \otimes_{\mathcal{B}} h^{n}\right]_{\mathcal{B}}\right)=\varphi_{n-1}\left(\left[h^{n} h^{0} \otimes_{\mathcal{B}} \cdots \otimes_{\mathcal{B}} h^{n-1}\right]_{\mathcal{B}}\right) \\
& =\left(\overline{h^{1_{(2)}} \cdots h^{n-1}{ }_{(2)}} \otimes \cdots \otimes \overline{h^{n-2}{ }_{(n-1)} h^{n-1_{(n-1)}}} \otimes \overline{h^{n-1}{ }_{(n)}} \otimes \overline{1}\right) \otimes_{\mathcal{H}} h^{n} h^{0} h_{(1)}^{1} \cdots h^{n-1_{(1)}} \\
& \stackrel{(3.5)}{=}\left(\overline{h^{1}{ }_{(2)} \cdots h^{n-1}(2)} \otimes \cdots \otimes \overline{h^{n-2}{ }_{(n-1)} h^{n-1}{ }_{(n-1)}} \otimes \overline{h^{n-1}(n)} \otimes \overline{1}\right) \\
& \otimes_{\mathcal{H}} h_{(2)}^{n} \triangleright\left(h^{0} h_{(1)}^{1} \cdots h^{n-1}{ }_{(1)} h_{(1)}^{n}\right) \\
& =\left(\overline{h^{1}{ }_{(2)} \cdots h^{n-1}{ }_{(2)}} \otimes \cdots \otimes \overline{h^{n-2}{ }_{(n-1)} h^{n-1}{ }_{(n-1)}} \otimes \overline{h^{n-1}{ }_{(n)}} \otimes \overline{1}\right) \cdot h_{(2)}^{n} \\
& \otimes_{\mathcal{H}} h^{0} h_{(1)}^{1} \cdots h^{n-1}{ }_{(1)} h_{(1)}^{n} \\
& =\left(\overline{h^{1}(2) \cdots h^{n}(2)} \otimes \cdots \otimes \overline{h^{n-1}{ }_{(n)} h^{n}(n)} \otimes \overline{h^{n}(n+1)}\right) \otimes_{\mathcal{H}} h^{0} h_{(1)}^{1} \cdots h^{n}{ }_{(1)} \\
& =\left(\overline{h_{(2)}^{1} \cdots h^{n}(2)} \otimes \cdots \otimes \overline{h^{n-1}{ }_{(n)} h_{(n)}^{n}} \otimes \overline{h_{(n+1)}^{n}} \otimes \varepsilon(\overline{1})\right) \otimes_{\mathcal{H}} h^{0} h_{(1)}^{1} \cdots h_{(1)}^{n} \\
& =d_{n} \varphi_{n}\left(\left[h^{0} \otimes_{\mathcal{B}} \cdots \otimes_{\mathcal{B}} h^{n}\right]_{\mathcal{B}}\right) \text {. }
\end{aligned}
$$


We next investigate the interaction with the degeneracy operators. To this end, for $0 \leq j \leq n-1$ we observe that

$$
\begin{aligned}
& \varphi_{n+1} s_{j}\left(\left[h^{0} \otimes_{\mathcal{B}} \cdots \otimes_{\mathcal{B}} h^{n}\right]_{\mathcal{B}}\right)=\varphi_{n+1}\left(\left[h^{0} \otimes_{\mathcal{B}} \cdots \otimes_{\mathcal{B}} h^{j} \otimes_{\mathcal{B}} 1 \otimes_{\mathcal{B}} h^{j+1} \otimes_{\mathcal{B}} \cdots \otimes_{\mathcal{B}} h^{n}\right]_{\mathcal{B}}\right) \\
& =\left(\overline{h^{1}(2) \cdots h^{n}(2)} \otimes \cdots \otimes \overline{h^{j}{ }_{(j+1)} \cdots h^{n}{ }_{(j+1)}} \otimes \overline{h^{j+1}{ }_{(j+2)} \cdots h^{n}(j+2)}\right. \\
& \otimes \overline{h^{j+1}{ }_{(j+3)} \ldots h^{n}{ }_{(j+3)}} \otimes \cdots \\
& \left.\otimes \overline{h^{n-1}(n+1)_{h^{n}}{ }_{(n+1)}} \otimes \overline{h^{n}(n+2)} \otimes \overline{1}\right) \otimes_{\mathcal{H}} h^{0} h^{1}{ }_{(1)} \cdots h^{n}{ }_{(1)} \\
& =\left(\overline{h^{1}(2) \cdots h^{n}(2)} \otimes \cdots \otimes \overline{h^{j}{ }_{(j+1)} \cdots h^{n}{ }_{(j+1)}} \otimes \Delta\left(\overline{h^{j+1}{ }_{(j+2)} \cdots h^{n}(j+2)}\right) \otimes \cdots\right. \\
& \left.\otimes \overline{h^{n-1}(n) h_{(n)}^{n}} \otimes \overline{h^{n}(n+1)} \otimes \overline{1}\right) \otimes \mathcal{H} h^{0} h^{1}{ }_{(1)} \cdots h_{(1)}^{n} \\
& =s_{j} \varphi_{n}\left(\left[h^{0} \otimes_{\mathcal{B}} \cdots \otimes_{\mathcal{B}} h^{n}\right]_{\mathcal{B}}\right) \text {. }
\end{aligned}
$$

Let us finally check the cyclic operators. To this end we have

$$
\begin{aligned}
& \varphi_{n} t_{n}\left(\left[h^{0} \otimes_{\mathcal{B}} \cdots \otimes_{\mathcal{B}} h^{n}\right]_{\mathcal{B}}\right)=\varphi_{n+1}\left(\left[h^{n} \otimes_{\mathcal{B}} h^{0} \otimes_{\mathcal{B}} \cdots \otimes_{\mathcal{B}} h^{n-1}\right]_{\mathcal{B}}\right) \\
& =\left(\overline{h^{0}(2) \cdots h^{n-1}{ }_{(2)}} \otimes \cdots \otimes \overline{h^{n-2}{ }_{(n)} h^{n-1}{ }_{(n)}} \otimes \overline{h^{n-1}{ }_{(n+1)}} \otimes \overline{1}\right) \otimes_{\mathcal{H}} h^{n} h_{(1)}^{0} \cdots h^{n-1}{ }_{(1)} \\
& =\left(\overline{h^{0}{ }_{(2)} h^{1_{(2)}} \cdots h^{n_{(2)}}} \otimes \overline{h^{1}{ }_{(3)} \cdots h^{n_{(3)}}} \otimes \cdots\right. \\
& \left.\otimes \overline{h^{n-1}(n+1)} h_{(n+1)}^{n} \otimes \overline{h^{n}(n+2)}\right) \otimes \mathcal{H}^{h_{(1)}} h_{(1)}^{1} \cdots h_{(1)}^{n} \\
& =\left(\overline{1} \cdot\left(h^{0} h_{(1)}^{1} \cdots h_{(1)}^{n}\right)_{(2)} \otimes \overline{h^{1}{ }_{(2)} \cdots h^{n}(2)} \otimes \cdots\right. \\
& \left.\otimes \overline{h^{n-1}{ }_{(n)} h_{(n)}^{n}} \otimes \overline{h_{(n+1)}^{n}}\right) \otimes_{\mathcal{H}}\left(h^{0} h^{1}{ }_{(1)} \cdots h_{(1)}^{n}\right)_{(1)} \\
& =t_{n} \varphi_{n}\left(\left[h^{0} \otimes_{\mathcal{B}} \cdots \otimes_{\mathcal{B}} h^{n}\right]_{\mathcal{B}}\right) \text {. }
\end{aligned}
$$

\subsection{Comparison with the Jara-Ştefan isomorphism in the homogeneous quotient-Hopf-Galois case}

In this subsection we compare the isomorphism of Theorem 3.4 with that of [27, Theorem 3.7] in the case of the homogeneous quotient Hopf-Galois extensions.

We note that in the case of $\mathcal{I}$ being a Hopf ideal of $\mathcal{H}$, analogously to (3.7) we have a map

$$
\begin{aligned}
& \bar{\varphi}_{n}:\left[\mathcal{H}^{\otimes_{\mathcal{B}} n+1}\right]_{\mathcal{B}} \cong(\mathcal{H} / \mathcal{I})^{\otimes n+1} \otimes_{\mathcal{H} / \mathcal{I}}[\operatorname{ad}(\mathcal{H})]_{\mathcal{B}} \\
& \bar{\varphi}_{n}\left(\left[h^{0} \otimes_{\mathcal{B}} \cdots \otimes_{\mathcal{B}} h^{n}\right]_{\mathcal{B}}\right) \\
& \quad=\left(\overline{h^{1}{ }_{(2)} \cdots h^{n}(2)} \otimes \cdots \otimes \overline{h^{n-1}{ }_{(n)} h^{n}{ }_{(n)}} \otimes \overline{h^{n}{ }_{(n+1)}} \otimes \overline{1}\right) \otimes_{\mathcal{H} / \mathcal{I}}\left[h^{0} h^{1}{ }_{(1)} \cdots h^{n}{ }_{(1)}\right]_{\mathcal{B}}
\end{aligned}
$$

which is well defined by the Hopf ideal assumption. It is easy to see that it is a special case of the isomorphism introduced by Jara-Ştefan [27, Theorem 3.7] originally in the context of Hopf-Galois extensions, when restricted to the context of homogeneous quotient-Hopf-Galois extensions.

Compared to (3.7), on the right hand side we tensorize over $\mathcal{H}$ from the right by $\operatorname{ad}(\mathcal{H})$ (with the left $\mathcal{H}$-module structure $(3.4))$ while in $(3.8)$ the tensor product by $[\operatorname{ad}(\mathcal{H})]_{\mathcal{B}}$ is over $\mathcal{H} / \mathcal{I}$ (with the left Miyashita-Ulbrich $\mathcal{H} / \mathcal{I}$-module structure).

However, in the case of homogeneous quotient-Hopf-Galois extensions the isomorphisms (3.7) and (3.8) coincide. Indeed, in this case $\mathcal{H} / \mathcal{I}$ is generated by $\overline{1}$ both as a left and a right $\mathcal{H}$ module, with respect to the multiplication from the left or from the right in $\mathcal{H}$. Accordingly, the degree zero component of the isomorphism (3.7) yields

$$
\varphi_{0}:[\mathcal{H}]_{\mathcal{B}} \stackrel{\cong}{\longrightarrow}(\mathcal{H} / \mathcal{I}) \otimes_{\mathcal{H}} \operatorname{ad}(\mathcal{H}), \quad \varphi_{0}\left([h]_{\mathcal{B}}\right)=\overline{1} \otimes_{\mathcal{H}} h,
$$


which is left $\mathcal{H} / \mathcal{I}$-linear with respect to the Miyashita-Ulbrich action on $[\mathcal{H}]_{\mathcal{B}}$ on the left hand side, and the algebra map $\mathcal{H} \rightarrow \mathcal{H} / \mathcal{I}$ on the right hand side, since

$$
\begin{aligned}
\varphi_{0}\left(\bar{h} \triangleright\left[h^{\prime}\right]_{\mathcal{B}}\right) & =\varphi_{0}\left(\left[h \triangleright h^{\prime}\right]_{\mathcal{B}}\right)=\overline{1} \otimes_{\mathcal{H}} h \triangleright h^{\prime}=\overline{1} \cdot h \otimes_{\mathcal{H}} \triangleright h^{\prime} \\
& =\bar{h} \otimes_{\mathcal{H}} h^{\prime}=h \cdot \overline{1} \otimes_{\mathcal{H}} h^{\prime}=h \cdot \varphi_{0}\left(\left[h^{\prime}\right]_{\mathcal{B}}\right) .
\end{aligned}
$$

We thus obtain an isomorphism of functors

$$
-\otimes_{\mathcal{H} / \mathcal{I}}[\operatorname{ad}(\mathcal{H})]_{\mathcal{B}} \cong-\otimes_{\mathcal{H} / \mathcal{I}}\left((\mathcal{H} / \mathcal{I}) \otimes_{\mathcal{H}} \operatorname{ad}(\mathcal{H})\right)=-\otimes_{\mathcal{H}} \operatorname{ad}(\mathcal{H})
$$

\subsection{A spectral sequence}

We note that the right hand side of Theorem 3.4, resp. the left hand side of Theorem 3.7, compute the homology of the algebra extension $\mathcal{H}$ relative to $\mathcal{B}$, resp. coextension $\mathcal{H}$ corelative to $\mathcal{C}$, while the other side computes the cyclic dual homology of the Pontryagin dual objects. In order to be able to investigate the latter homologies, in this subsection we shall develop computational tools.

We will focus on the Hochschild homology groups of the relative homology of the extension.

Theorem 3.5. Let $\mathcal{I} \subseteq \mathcal{H}$ be a coideal right ideal in a Hopf algebra $\mathcal{H}$ such that $\mathcal{H}^{\mathrm{co}} \mathcal{H} / \mathcal{I} \subseteq \mathcal{H}$ be a homogeneous $\mathcal{H} / \mathcal{I}$-Galois extension. Then there exists a spectral sequence (constructed in the proof) such that

$$
\mathrm{HH}_{\bullet}\left(\mathcal{H} \mid \mathcal{H}^{\mathrm{co} \mathcal{H} / \mathcal{I}}\right)=\mathrm{E}_{\bullet}^{2}, 0, \quad \mathrm{E}_{\bullet, \bullet}^{2} \Longrightarrow \operatorname{Tor}_{\bullet}^{\mathcal{H}}(k, \operatorname{ad}(\mathcal{H}))
$$

In particular, we have a five-term exact sequence

$$
\begin{aligned}
\operatorname{Tor}_{2}^{\mathcal{H}}(k, \operatorname{ad}(\mathcal{H})) & \rightarrow \mathrm{HH}_{2}\left(\mathcal{H} \mid \mathcal{H}^{\operatorname{co} \mathcal{H} / \mathcal{I}}\right) \rightarrow \mathrm{H}_{0}\left(\operatorname{Tor}_{1}^{\mathcal{H}}\left((\mathcal{H} / \mathcal{I})^{\otimes \bullet+1}, \operatorname{ad}(\mathcal{H})\right)\right) \rightarrow \cdots \\
\cdots \rightarrow \operatorname{Tor}_{1}^{\mathcal{H}}(k, \operatorname{ad}(\mathcal{H})) & \rightarrow \mathrm{HH}_{1}\left(\mathcal{A} \mid \mathcal{H}^{\operatorname{co} \mathcal{H} / \mathcal{I}}\right) \rightarrow 0 .
\end{aligned}
$$

Proof. Let us consider the cyclic dual $\mathcal{C}$. to the standard cocyclic object of the coalgebra $\mathcal{C}:=\mathcal{H} / \mathcal{I}$, consisting of the tensor powers of $\mathcal{C}$. More precisely, $\mathcal{C}_{p}=\mathcal{C}{ }^{\otimes p+1}$, with the boundary map $\partial: \mathcal{C}_{p} \rightarrow \mathcal{C}_{p-1}$ being

$$
\partial\left(c^{0} \otimes \cdots \otimes c^{p}\right)=\sum_{i=0}^{p}(-1)^{i} c^{0} \otimes \cdots \otimes \varepsilon\left(c^{i}\right) c^{i+1} \otimes \cdots \otimes c^{p},
$$

where the cyclic order of length $p+1$ is assumed. Note that $\partial$ is a morphism of right $\mathcal{H}$-modules. The operator $h: \mathcal{C}_{p} \rightarrow \mathcal{C}_{p+1}$

$$
h\left(c^{0} \otimes \cdots \otimes c^{p}\right):=\overline{1} \otimes c^{0} \otimes \cdots \otimes c^{p}
$$

is a homotopy contracting this complex to $k$ concentrated at zero degree, see for instance [31]. Let $\mathcal{M}$. be a flat resolution of the left $\mathcal{H}$-module $\mathcal{M}:=\operatorname{ad}(\mathcal{H})$, and consider the total complex $\mathcal{C} \bullet \otimes_{\mathcal{H}} \mathcal{M}_{\bullet}$. We have two spectral sequences abutting to the total homology. The first page of the first one reads as

$$
\mathrm{E}_{p, q}^{1}=\mathrm{H}_{q}\left(\mathcal{C}_{p} \otimes_{\mathcal{H}} \mathcal{M}_{\bullet}\right)=\operatorname{Tor}_{q}^{\mathcal{H}}\left(\mathcal{C}_{p}, \mathcal{M}\right),
$$

hence its second page is computed as

$$
\mathrm{E}_{p, q}^{2}=\mathrm{H}_{p}\left(\operatorname{Tor}_{q}^{\mathcal{H}}\left(\mathcal{C}_{\bullet}, \mathcal{M}\right)\right)
$$


By Theorem 3.4, we are interested in

$$
\mathrm{HH}_{n}\left(\mathcal{H} \mid \mathcal{H}^{\mathrm{co} \mathcal{H} / \mathcal{I}}\right)=\mathrm{E}_{n, 0}^{2} .
$$

Now, let us compute the first page of the second spectral sequence, the transposed analogue of the first one, abutting to the total homology, which by flatness of the resolution $\mathcal{M}_{\bullet}$ and the acyclicity of $\mathcal{C}$. can be rewritten as

$$
{ }^{\top} \mathrm{E}_{p, q}^{1}=\mathrm{H}_{p}\left(\mathcal{C}_{\bullet} \otimes_{\mathcal{H}} \mathcal{M}_{q}\right)=\mathrm{H}_{p}\left(\mathcal{C}_{\bullet}\right) \otimes_{\mathcal{H}} \mathcal{M}_{q}=\left\{\begin{array}{lll}
k \otimes_{\mathcal{H}} \mathcal{M}_{q} & \text { for } & p=0 \\
0 & \text { for } & p>0
\end{array}\right.
$$

As a result, degenerating at the second page,

$$
{ }^{\top} \mathrm{E}_{p, q}^{2}=\left\{\begin{array}{lll}
\operatorname{Tor}_{q}^{\mathcal{H}}(k, \mathcal{M}) & \text { for } & p=0 \\
0 & \text { for } & p>0
\end{array}\right.
$$

this spectral sequence yields the total cohomology

$$
\mathrm{H}_{n}\left(\mathcal{C} \bullet \otimes_{\mathcal{H}} \mathcal{M}_{\bullet}\right)=\operatorname{Tor}_{n}^{\mathcal{H}}(k, \mathcal{M})
$$

Finally, we use (3.9), (3.10) and the canonical homological five-term exact sequence

$$
\mathrm{H}_{2} \rightarrow \mathrm{E}_{2,0}^{2} \stackrel{d}{\rightarrow} \mathrm{E}_{0,1}^{2} \rightarrow \mathrm{H}_{1} \rightarrow \mathrm{E}_{1,0}^{2} \rightarrow 0
$$

to finish the proof. The second arrow is the boundary map of the second page of the first spectral sequence, and the next arrow is induced by the augmentation $\mathcal{C}_{\bullet} \rightarrow k$.

Next we show that the above theorem generalizes the classical result from the case of the homogeneous $\mathcal{H}$-Galois extension $k \subseteq \mathcal{H}$ to arbitrary homogeneous quotient coalgebra-Galois extensions $\mathcal{B} \subseteq \mathcal{H}$. The following can be regarded as an independent proof of this classical result.

Corollary 3.6. For any Hopf algebra $\mathcal{H}$,

$$
\mathrm{HH}_{\bullet}(\mathcal{H})=\operatorname{Tor}_{\bullet}^{\mathcal{H}}(k, \operatorname{ad}(\mathcal{H})),
$$

where on the right hand side the left $\mathcal{H}$-module structure on $\mathcal{H}$ comes from its canonical SAYD module structure.

Proof. Since for every right $\mathcal{H}$-module $\mathcal{N}$ the invertible linear map

$$
\mathcal{N} \otimes \mathcal{H} \longrightarrow \mathcal{N} \otimes \mathcal{H}, \quad n \otimes h \mapsto n S\left(h_{(1)}\right) \otimes h_{(2)}
$$

makes the diagonal right $\mathcal{H}$-module $\mathcal{N} \otimes \mathcal{H}$ free, hence flat, then by induction the diagonal right $\mathcal{H}$-module $\mathcal{C}_{\bullet}=\mathcal{H}^{\otimes \bullet+1}$ is flat. This implies that

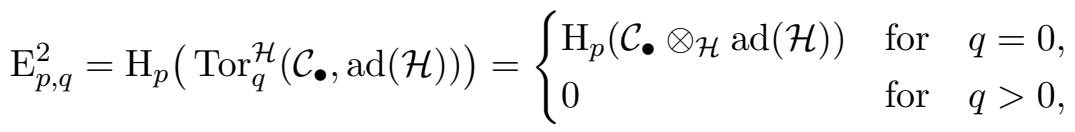

hence the first spectral sequence degenerates at the second page as well, and therefore we obtain

$$
\mathrm{HH}_{n}(\mathcal{H})=\mathrm{HH}_{n}(\mathcal{H} \mid k) \stackrel{(3.9)}{=} \mathrm{E}_{n, 0}^{2} \stackrel{(3.12)}{=} \mathrm{H}_{n}\left(\mathcal{C}_{\bullet} \otimes_{\mathcal{H}} \mathcal{M}_{\bullet}\right) \stackrel{(3.10)}{=} \operatorname{Tor}_{n}^{\mathcal{H}}(k, \mathcal{H})
$$




\subsection{The isomorphism of Pontryagin duals}

In this subsection, we discuss the Pontryagin dual of the results of the previous subsection (we will call the results of Subsection 3.1 direct picture, and their dual counterparts dual picture) in detail. Hence, the result of the present subsection can be interpreted as the cyclic-homological dual Takeuchi-Galois transform accompanying the Takeuchi-Galois transform $\mathcal{B} \mapsto \mathcal{H} / \mathcal{B}^{+} \mathcal{H}$. More explicitly, we shall obtain an isomorphism between the Hopf-cyclic homology of the $\mathcal{H}$ comodule algebra $\mathcal{B}$ and the relative homology of a $\mathcal{B}$-Galois coextension $\mathcal{D}:=\mathcal{H} \rightarrow \mathcal{H} / \mathcal{B}^{+} \mathcal{H}=: \mathcal{C}$ mentioned in the following theorem.

Theorem 3.7. Let $\mathcal{B} \subseteq \mathcal{H}$ be a left comodule subalgebra in a Hopf algebra $\mathcal{H}$ such that $\mathcal{H} \rightarrow$ $\mathcal{H} / \mathcal{B}^{+} \mathcal{H}$ is a $\mathcal{B}-$ Galois coextension, and $\operatorname{coad}(\mathcal{H})=\mathcal{H}$ be the right-left SAYD module with the right action given by the multiplication of $\mathcal{H}$, and the left coadjoint coaction. Then there exists an isomorphism

$$
\gamma_{n}: \mathrm{C}_{n}(\mathcal{B}, \operatorname{coad}(\mathcal{H}))^{\mathcal{H}} \longrightarrow \mathrm{C}_{n}\left(\mathcal{H} \mid \mathcal{H} / \mathcal{B}^{+} \mathcal{H}\right)
$$

of cyclic modules, defined by (3.13), (3.14).

Proof. We note that the proof of Theorem 3.4 uses only the structure maps and the relations equivalent to the commutativity of appropriate diagrams, therefore, is in fact diagrammatical. Then applying the formal Pontryagin duality by reversing the arrows, interchanging the left and right, next applying the cyclic duality and finally inverting the resulting isomorphism yields a diagrammatical proof of the claim. More precisely, the isomorphism is given by

$$
\begin{aligned}
& \gamma_{n}: \operatorname{coad}(\mathcal{H}) \square_{\mathcal{H}} \mathcal{B}^{\otimes n+1} \longrightarrow(\underbrace{\mathcal{H} \square_{\mathcal{C}} \mathcal{H} \square_{\mathcal{C}} \cdots \square_{\mathcal{C}} \mathcal{H}}_{n+1 \text { many }})^{\mathcal{C}}, \\
& h \otimes b^{0} \otimes \cdots \otimes b^{n} \mapsto b^{1}{ }_{(2)} \cdots b^{n}{ }_{(2)} h_{(2)} \otimes \cdots \otimes b^{n}{ }_{(n+1)} h_{(n+1)} \otimes b^{0} b^{1}{ }_{(1)} \cdots b^{n}{ }_{(1)} h_{(1)},
\end{aligned}
$$

and

$$
\begin{aligned}
& \gamma_{n}^{-1}:(\underbrace{\mathcal{H} \square_{\mathcal{C}} \mathcal{H} \square_{\mathcal{C}} \cdots \square_{\mathcal{C}} \mathcal{H}}_{n+1 \text { many }})^{\mathcal{C}} \longrightarrow \operatorname{coad}(\mathcal{H}) \square_{\mathcal{H}} \mathcal{B}^{\otimes n+1}, \\
& h^{0} \otimes \cdots \otimes h^{n} \mapsto h^{n}{ }_{(2)} \otimes h^{n}{ }_{(3)} S\left(h^{0}{ }_{(1)}\right) \otimes h_{(2)}^{0} S\left(h^{1}{ }_{(1)}\right) \otimes \cdots \otimes h^{n-1}{ }_{(2)} S\left(h^{n}{ }_{(1)}\right) .
\end{aligned}
$$

We next show that the cyclic structure on $C_{\bullet}(\mathcal{C} \mid \mathcal{H})$ corresponds, via the isomorphisms $(3.13)$ and (3.14), to the Hopf-cyclic structure on $C_{\bullet}(\mathcal{B}, \operatorname{coad}(\mathcal{H}))^{\mathcal{H}}$.

We first note for $0 \leq i \leq n-1$ that

$$
\begin{aligned}
& \gamma_{n-1} \circ d_{i}\left(h \otimes b^{0} \otimes \cdots \otimes b^{n}\right)=\gamma_{n-1}\left(h \otimes b^{0} \otimes \cdots \otimes b^{i} b^{i+1} \otimes \cdots \otimes b^{n}\right) \\
& =b_{(2)}^{1_{(2)}} \cdots b_{(2)}^{n} h_{(2)} \otimes \cdots \otimes b_{(i+1)}^{i} b^{i+1}{ }_{(i+1)} b^{i+2}{ }_{(i+1)} \cdots b_{(i+1)}^{n} h_{(i+1)} \\
& \otimes b^{i+2}{ }_{(i+2)} \cdots b_{(i+2)}^{n} h_{(i+2)} \otimes \cdots \otimes b_{(n)}^{n} h_{(n)} \otimes b^{0} b_{(1)}^{1} \cdots b_{(1)}^{n} h_{(1)} \\
& =b_{(2)}^{1_{(2)}} \cdots b_{(2)}^{n_{(2)}} h_{(2)} \otimes \otimes b_{(i+1)}^{i_{(i+1)}} b^{i+2}{ }_{(i+1)} \cdots b_{(i+1)}^{n_{(i+1)}} \\
& \otimes \varepsilon\left(b^{i+1_{(i+2)}} b^{i+2}{ }_{(i+2)} \cdots b_{(i+2)}^{n} h_{(i+2)}\right) \otimes b^{i+2}{ }_{(i+3)} \cdots b_{(i+3)}^{n} h_{(i+3)} \otimes \cdots \\
& \otimes b_{(n+1)}^{n} h_{(n+1)} \otimes b^{0} b_{(1)}^{1} \cdots b_{(1)}^{n} h_{(1)} \\
& =d_{i} \circ \gamma_{n}\left(h \otimes b^{0} \otimes \cdots \otimes b^{n}\right) \text {. }
\end{aligned}
$$

As for $i=n$, we have

$$
\begin{aligned}
& \gamma_{n-1} \circ d_{n}\left(h \otimes b^{0} \otimes \cdots \otimes b^{n}\right)=\gamma_{n-1}\left(b_{(1)}^{n} h \otimes b_{(2)}^{n} b^{0} \otimes \cdots \otimes b^{n-1}\right) \\
& =b_{(2)}^{1_{(2)}} \cdots b^{n-1_{(2)}} b_{(1)(2)}^{n} h_{(2)} \otimes \cdots \otimes b_{(n)}^{n-1_{(1)(n)}} h_{(n)} \otimes b_{(2)}^{n} b^{0} b_{(1)}^{1_{(1)}} \cdots b_{(1)}^{n} h_{(1)}
\end{aligned}
$$




$$
\begin{aligned}
& =b_{(2)}^{1_{(2)}} \cdots b_{(2)}^{n} h_{(2)} \otimes \cdots \otimes b^{n-1}{ }_{(n)} b_{(n)}^{n} h_{(n)} \otimes b_{(2)}^{n} b^{0} b_{(1)}^{1} \cdots b_{(1)}^{n} h_{(1)} \\
& =b_{(1)}^{1} \cdots b_{(1)}^{n} h_{(2)(2)} \otimes \cdots \otimes b_{(n-1)}^{n-1} b_{(n-1)}^{n} h_{(2)(n)} \otimes b_{(n)}^{n} h_{(3)} S\left(h_{(1)}\right) h_{(2)(1)} \varepsilon\left(b^{0}\right) \\
& =b_{(1)}^{1} \cdots b_{(1)}^{n} h_{(1)} \otimes \cdots \otimes b^{n-1}{ }_{(n-1)} b_{(n-1)}^{n} h_{(n-1)} \otimes b_{(n)}^{n} h_{(n)} \varepsilon\left(b^{0}\right) \\
& =b_{(2)}^{1} \cdots b_{(2)}^{n_{(2)}} h_{(2)} \otimes \otimes b_{(n+1)}^{n} h_{(n+1)} \otimes \varepsilon\left(b^{0} b_{(1)}^{1} \cdots b_{(1)}^{n} h_{(1)}\right) \\
& =d_{n} \circ \gamma_{n}\left(h \otimes b^{0} \otimes \cdots \otimes b^{n}\right) \text {, }
\end{aligned}
$$

where on the third equality we used

$$
h \otimes b_{(1)}^{0} \cdots b_{(1)}^{n} \otimes \varepsilon\left(b_{(2)}^{0}\right) \otimes \cdots \otimes b_{(2)}^{n}=h_{(2)} \otimes h_{(3)} S\left(h_{(1)}\right) \otimes \varepsilon\left(b^{0}\right) \otimes \cdots \otimes b^{n} .
$$

We next note that

$$
\begin{aligned}
& \gamma_{n+1} \circ s_{j}\left(h \otimes b^{0} \otimes \cdots \otimes b^{n}\right)=\gamma_{n+1}\left(h \otimes \otimes b^{0} \otimes \cdots \otimes b^{j} \otimes 1 \otimes \cdots \otimes b^{n}\right) \\
& =b_{(2)}^{1_{(2)}} \cdots b_{(2)}^{n} h_{(2)} \otimes \cdots \otimes b^{j+1_{(j+1)}} \cdots b_{(j+1)}^{n_{(j+1)}} \otimes b^{j+1_{(j+2)}} \cdots b_{(j+2)}^{n_{(j+2)}} h_{(j+\cdots} \\
& \otimes b_{(n+1)}^{n} h_{(n+1)} \otimes b^{0} b^{1}{ }_{(1)} \cdots b_{(1)}^{n} h_{(1)} \\
& =b_{(2)}^{1} \cdots b_{(2)}^{n} h_{(2)} \otimes \cdots \otimes \Delta\left(b^{j+1}{ }_{(j+1)} \cdots b_{(j+1)}^{n} h_{(j+1)}\right) \otimes \cdots \\
& \otimes b_{(n+1)}^{n} h_{(n+1)} \otimes b^{0} b_{(1)}^{1} \cdots b_{(1)}^{n} h_{(1)} \\
& =s_{j} \circ \gamma_{n}\left(h \otimes b^{0} \otimes \cdots \otimes b^{n}\right) \text {. }
\end{aligned}
$$

Finally we observe that

$$
\begin{aligned}
& \gamma_{n} \circ t_{n}\left(h \otimes b^{0} \otimes \cdots \otimes b^{n}\right)=\gamma_{n}\left(b_{(1)}^{n} h \otimes b_{(2)}^{n} \otimes b^{0} \otimes \cdots \otimes b^{n-1}\right) \\
& =b_{(2)}^{0} \cdots b^{n-1}{ }_{(2)} b_{(1)(2)}^{n} h_{(2)} \otimes \cdots \otimes b^{n-1}{ }_{(n+1)} b_{(1)(n)}^{n} h_{(n+1)} \\
& \otimes b^{n_{(2)}} b_{(1)}^{0} \cdots b^{n-1}{ }_{(1)} b_{(1)(1)}^{n} h_{(1)} \\
& =b_{(2)}^{0} \cdots b_{(2)}^{n_{(2)}} h_{(2)} \otimes \otimes b^{n-1_{(n+1)}} b_{(n+1)}^{n_{(n+1)}} \otimes b_{(n+2)}^{n_{(1)}} b_{(1)}^{0} \cdots b^{n-1_{(1)}} b_{(1)}^{n_{(1)}} h_{(1)} \\
& =b^{0} b_{(1)}^{1} \cdots b_{(1)}^{n} h_{(2)(2)} \otimes \cdots \otimes b_{(n)}^{n-1} b_{(n)}^{n} h_{(2)(n+1)} \otimes b_{(n+1)}^{n} h_{(3)} S\left(h_{(1)}\right) h_{(2)(1)} \\
& =b^{0} b_{(1)}^{1} \cdots b_{(1)}^{n} h_{(1)} \otimes b_{(2)}^{1} \cdots b_{(2)}^{n} h_{(2)} \otimes \cdots \otimes b^{n}{ }_{(n+1)} h_{(n+1)} \\
& =t_{n} \circ \gamma_{n}\left(h \otimes b^{0} \otimes \cdots \otimes b^{n}\right) \text {. }
\end{aligned}
$$

\subsection{The classical case}

For any affine scheme $X$ over a field $k$ we will denote by $\mathscr{O}(X)$ the $k$-algebra of regular functions on $X$. Assume that $G$ is a linear algebraic group over a field $k$ and $H$ its closed algebraic subgroup. Assume that both are linearly reductive, hence all quotients below exist as affine varieties.

\subsubsection{The direct picture in the classical case}

The direct picture for $\mathcal{H}=\mathscr{O}(G), \mathcal{C}=\mathscr{O}(H), \mathcal{B}=\mathcal{H}^{\text {co } \mathcal{C}}=\mathscr{O}(G / H)$, simplifies a lot because of the commutativity of $\mathcal{H}$. Since $\mathcal{B}$ is central in $\mathcal{H}$, the cyclic tensor power $\left[\mathcal{H}^{\otimes_{\mathcal{B}} n+1}\right]_{\mathcal{B}}$ coincides with the usual tensor power $\mathcal{H}^{\otimes_{\mathcal{B}} n+1}$. Moreover, the left $\operatorname{SAYD} \mathcal{H}$-action on $\operatorname{ad}(\mathcal{H})$ factors through the counit, and hence we can replace $\operatorname{ad}(\mathcal{H})$ by $\mathcal{H}$ regarded as a trivial $\mathcal{H}$-module.

The right diagonal $\mathcal{H}$-action on the tensor power of $\mathcal{H} / \mathcal{I}$ comes from the multiplication in $G$ of elements of the subgroup $H$. Moreover, both sides become $k$-algebras and the isomorphism (3.7) becomes an algebra map

$$
\varphi_{n}: \mathcal{H}^{\otimes \mathcal{B} n+1} \stackrel{\cong}{\longrightarrow}\left((\mathcal{H} / \mathcal{I})^{\otimes n+1} \otimes_{\mathcal{H}} k\right) \otimes \mathcal{H},
$$


which describes a map of algebraic varieties

$$
\begin{aligned}
& G^{\times_{G / H}{ }^{n+1}} \longleftarrow\left(H^{n+1} \times_{G} \mathrm{pt}\right) \times G, \\
& \left(g, g h_{0}, g h_{0} h_{1}, \ldots, g h_{0} \ldots h_{n-1}\right) \leftarrow\left(h_{0}, \ldots, h_{n} ; g\right),
\end{aligned}
$$

with the inverse

$$
\begin{aligned}
& G^{\times_{G / H}{ }^{n+1}} \longrightarrow\left(H^{n+1} \times_{G} \mathrm{pt}\right) \times G, \\
& \left(g_{0}, \ldots, g_{n}\right) \mapsto\left(g_{0}^{-1} g_{1}, g_{1}^{-1} g_{2}, \ldots, g_{n}^{-1} g_{0} ; g_{0}\right) .
\end{aligned}
$$

The left hand side is the fiber power of the canonical projection $G \rightarrow G / H$ consisting of tuples $\left(g_{0}, \ldots, g_{n}\right)$ such that $g_{0} H=\cdots=g_{n} H$. These varieties form a cocyclic variety with the coface maps

$$
\delta_{i}: G^{\times / H}{ }^{n} \longrightarrow G^{\times_{G / H}}{ }^{n+1}, \quad \delta_{i}\left(g_{0}, \ldots, g_{n}\right)=\left(g_{0}, \ldots, g_{i}, g_{i}, \ldots, g_{n}\right),
$$

the codegeneracy maps

$$
\sigma_{i}: G^{\times_{G / H}}{ }^{n+1} \longrightarrow G^{\times_{G / H}}{ }^{n}, \quad \sigma_{i}\left(g_{0}, \ldots, g_{n}\right)=\left(g_{0}, \ldots, g_{i-1}, g_{i+1}, \ldots, g_{n}\right),
$$

and the cocyclic map

$$
\tau_{n}: G^{\times_{G / H}}{ }^{n+1} \longrightarrow G^{\times_{G / H}}{ }^{n+1}, \quad \tau_{n}\left(g_{0}, g_{1}, \ldots, g_{n}\right)=\left(g_{1}, \ldots, g_{n}, g_{0}\right) .
$$

We note that this is the cyclic dual of the Cech nerve of the orbital map $G \rightarrow G / H$.

The right hand side is the cartesian product of the fiber product $H^{n+1} \times_{G}$ pt of the map $H^{n+1} \rightarrow G,\left(h_{0}, \ldots, h_{n}\right) \mapsto h_{0} \cdots h_{n}$ and the map pt $\rightarrow G, \star \mapsto e$, consisting of tuples $\left(h_{0}, \ldots, h_{n}\right)$ such that $h_{0} \cdots h_{n}=e$, and $G$. The cocyclic variety formed by these varieties has the coface maps

$$
\begin{aligned}
& \delta_{i}:\left(H^{n} \times_{G} \mathrm{pt}\right) \times G \longrightarrow\left(H^{n+1} \times_{G} \mathrm{pt}\right) \times G, \\
& \delta_{i}\left(h_{0}, \ldots, h_{n-1} ; g\right)=\left(h_{0}, \ldots, h_{i-1}, e, h_{i}, \ldots, h_{n-1} ; g\right),
\end{aligned}
$$

the codegeneracy maps

$$
\begin{aligned}
& \sigma_{i}:\left(H^{n+1} \times_{G} \mathrm{pt}\right) \times G \longrightarrow\left(H^{n} \times_{G} \mathrm{pt}\right) \times G, \\
& \sigma_{i}\left(h_{0}, \ldots, h_{n} ; g\right)=\left(h_{0}, \ldots, h_{i} h_{i+1}, \ldots, h_{n} ; g\right),
\end{aligned}
$$

and the cocyclic map

$$
\begin{aligned}
& \tau_{n}:\left(H^{n+1} \times_{G} \mathrm{pt}\right) \times G \longrightarrow\left(H^{n+1} \times_{G} \mathrm{pt}\right) \times G, \\
& \tau_{n}\left(h_{0}, h_{1}, \ldots, h_{n} ; g\right)=\left(h_{1}, \ldots, h_{n}, h_{0} ; g h_{0}\right) .
\end{aligned}
$$

It is easy to check that the maps (3.15), (3.16) are mutually inverse isomorphisms of cocyclic schemes.

Remark 3.8. It is worth explaining the classical meaning of the isomorphism (3.11). Let $\mathcal{H}$ be the Hopf algebra $\mathscr{O}(G)$ of regular functions on a linear algebraic group $G$ over a field $k$ of characteristic zero, $\mathfrak{g}$ the Lie algebra of $G$, and $\mathfrak{m} \subset \mathscr{O}(G)$ the maximal ideal at the neutral element.

The left hand side of (3.11) can be computed by the Hochschild-Kostant-Rosenberg theorem [26] as the vector space of regular differential forms on $G$,

$$
\mathrm{HH}_{\bullet}(\mathcal{H})=\Omega^{\bullet}(G) \text {. }
$$


As for the right hand side we use the commutativity of $\mathscr{O}(G)$. Then the left $\mathscr{O}(G)$-module structure on $\operatorname{ad}(\mathscr{O}(G))$, a priori coming from the left-right SAYD-module structure, factors through the trivial action on $k$ by the counit. From geometric point of view, the counit is simply the evaluation at the neutral element of $G$, corresponding to the maximal ideal $\mathfrak{m} \subseteq \mathscr{O}(G)$. Then we can use the identification $k=\mathscr{O}(G) / \mathfrak{m}$, and the regularity of the group variety at the neutral element, to use Serre's formula

$$
\operatorname{Tor}_{\bullet}^{R}(R / \mathfrak{m}, R / \mathfrak{m})=\bigwedge_{R / \mathfrak{m}}^{\bullet}\left(\mathfrak{m} / \mathfrak{m}^{2}\right),
$$

where $R=\mathscr{O}(G)_{\mathfrak{m}}$ is the local ring at the neutral element.

Using finally the identification $\mathfrak{m} / \mathfrak{m}^{2}=\mathfrak{g}^{*}$, we obtain on the right hand side of (3.11),

$$
\operatorname{Tor}_{\bullet}^{\mathcal{H}}(k, \mathcal{H})=\operatorname{Tor}_{\bullet}^{\mathscr{O}(G)}(k, \mathscr{O}(G))=\operatorname{Tor}_{\bullet}^{\mathscr{O}(G)}(k, k) \otimes \mathscr{O}(G)=\bigwedge^{\bullet} \mathfrak{g}^{*} \otimes \mathscr{O}(G) .
$$

Since both sides of (3.11) are $\mathscr{O}(G)$-modules and the isomorphism is $\mathscr{O}(G)$-linear, (3.11) reads simply as triviality of the bundle of regular forms on a linear algebraic group $G$

$$
\Omega^{\bullet}(G)=\bigwedge^{\bullet} \mathfrak{g}^{*} \otimes \mathscr{O}(G)
$$

\subsubsection{The dual picture in the classical case}

The dual picture for $\mathcal{H}=\mathscr{O}(G), \mathcal{C}=\mathscr{O}(H), \mathcal{B}=\mathcal{H}^{\text {co } \mathcal{C}}=\mathscr{O}(G / H)$, also can be made an isomorphism of cocyclic affine schemes over $k$, however more interesting, especially if $\mathcal{H}$ is not cocommutative, or equivalently, if $G$ is not abelian. Then the left-right SAYD module coad $(\mathcal{H})$ can be identified with $\mathscr{O}(\operatorname{ad}(G))$, where $\operatorname{ad}(G)$ coincides with $G$ as a variety, the left $\mathcal{H}$-module structure comes from a diagonal map of $G$, and the right $\mathcal{H}$-comodule structure comes from the right action of $G$ on itself by conjugations. Below it will be more convenient to pass to the left diagonal action of $G$ on $\operatorname{ad}(G) \times(G / H)^{n+1}$ with the use of the equivalent left $G$-action on $\operatorname{ad}(G)$ by conjugations. The isomorphism of the cyclic modules given by the maps

$$
\operatorname{coad}(\mathcal{H}) \square_{\mathcal{H}}\left(\mathcal{B}^{\otimes n+1}\right) \longrightarrow\left(\mathcal{H}^{\square_{\mathcal{C}}(n+1)}\right)^{\mathcal{C}}
$$

reads now as an isomorphism of cocyclic varieties

$$
G^{n+1} / H^{n+1} \longrightarrow G \backslash\left(\operatorname{ad}(G) \times(G / H)^{n+1}\right) .
$$

On the left hand side we quotient by the right $H^{n+1}$-action

$$
\begin{aligned}
& G^{n+1} \times H^{n+1} \longrightarrow G^{n+1}, \\
& \left(g_{0}, \ldots, g_{n}\right) \cdot\left(h_{0}, \ldots, h_{n}\right):=\left(h_{0}^{-1} g_{0} h_{1}, h_{1}^{-1} g_{1} h_{2}, \ldots, h_{n}^{-1} g_{n} h_{0}\right) .
\end{aligned}
$$

Note that this action is not free. The stabilizer of $\left(g_{0}, \ldots, g_{n}\right)$ consists of the $(n+1)$-tuples $\left(h_{0}, \ldots, h_{n}\right)$ such that

$$
\begin{aligned}
& h_{0} \in \mathrm{C}_{G}\left(g_{0} \cdots g_{n}\right) \cap \bigcap_{i=0}^{n}\left(g_{0} \cdots g_{i}\right) H\left(g_{0} \cdots g_{i}\right)^{-1}, \\
& h_{i}=\left(g_{0} \cdots g_{i-1}\right) h_{0}\left(g_{0} \cdots g_{i-1}\right)^{-1} \quad \text { for } \quad i=1, \ldots, n .
\end{aligned}
$$

The cocyclic structure on the left hand side is given by the coface operators

$$
\delta_{i}: G^{n+1} / H^{n+1} \longrightarrow G^{n+2} / H^{n+2}, \quad \delta_{i}\left[g_{0}, g_{1}, \ldots, g_{n}\right]=\left[g_{0}, \ldots, g_{i-1}, e, g_{i}, \ldots, g_{n}\right]
$$


the codegeneracy operators

$$
\sigma_{i}: G^{n+1} / H^{n+1} \longrightarrow G^{n} / H^{n}, \quad \sigma_{i}\left[g_{0}, g_{1}, \ldots, g_{n}\right]=\left[g_{0}, \ldots, g_{i} g_{i+1}, \ldots, g_{n}\right],
$$

and the cocyclic operator

$$
\tau_{n}: G^{n+1} / H^{n+1} \longrightarrow G^{n+1} / H^{n+1}, \quad \tau_{n}\left[g_{0}, g_{1}, \ldots, g_{n}\right]=\left[g_{1}, \ldots, g_{n}, g_{0}\right] .
$$

On the right hand side we quotient by the left $G$-action

$$
\begin{aligned}
& G \times\left(\operatorname{ad}(G) \times(G / H)^{n+1}\right) \longrightarrow \operatorname{ad}(G) \times(G / H)^{n+1}, \\
& g \cdot\left(\widetilde{g}, g_{0} H, \ldots, g_{n} H\right):=\left(g \widetilde{g} g^{-1}, g g_{0} H, \ldots, g g_{n} H\right) .
\end{aligned}
$$

Note that this action is not free as well. The stabilizer of $\left(\widetilde{g}, g_{0} H, \ldots, g_{n} H\right)$ consists of the elements

$$
g \in \mathrm{C}_{G}(\widetilde{g}) \cap \bigcap_{i=0}^{n} g_{i} H g_{i}^{-1} .
$$

The cocyclic structure on the right hand side is given by the coface operators

$$
\begin{aligned}
& \delta_{i}: G \backslash\left(\operatorname{ad}(G) \times(G / H)^{n+1}\right) \longrightarrow G \backslash\left(\operatorname{ad}(G) \times(G / H)^{n+2}\right), \\
& \delta_{i}\left[\widetilde{g}, g_{0} H, \ldots, g_{n} H\right]=\left[\widetilde{g}, g_{0} H, \ldots, g_{i} H, g_{i} H, \ldots, g_{n} H\right]
\end{aligned}
$$

the codegeneracy operators

$$
\begin{aligned}
& \sigma_{i}: G \backslash\left(\operatorname{ad}(G) \times(G / H)^{n+1}\right) \longrightarrow G \backslash\left(\operatorname{ad}(G) \times(G / H)^{n}\right), \\
& \sigma_{i}\left[\widetilde{g}, g_{0} H, \ldots, g_{n} H\right]=\left[\widetilde{g}, g_{0} H, \ldots, g_{i-1} H, g_{i+1} H, \ldots, g_{n} H\right],
\end{aligned}
$$

and the cocyclic operator

$$
\begin{aligned}
& \tau_{n}: G \backslash\left(\operatorname{ad}(G) \times(G / H)^{n+1}\right) \longrightarrow G \backslash\left(\operatorname{ad}(G) \times(G / H)^{n+1}\right), \\
& \tau_{n}\left[\widetilde{g}, g_{0} H, \ldots, g_{n} H\right]=\left[\widetilde{g}, g_{1} H, \ldots, g_{n} H, \widetilde{g} g_{0} H\right] .
\end{aligned}
$$

It follows readily that the maps

$$
\begin{aligned}
& G^{n+1} / H^{n+1} \longrightarrow G \backslash\left(\operatorname{ad}(G) \times(G / H)^{n+1}\right), \\
& {\left[g_{0}, g_{1}, \ldots, g_{n}\right] \mapsto\left[g_{0} g_{1} \cdots g_{n} ; g_{0} H, g_{0} g_{1} H, \ldots, g_{0} g_{1} \cdots g_{n} H\right]}
\end{aligned}
$$

and

$$
\begin{aligned}
& G^{n+1} / H^{n+1} \longleftarrow G \backslash\left(\operatorname{ad}(G) \times(G / H)^{n+1}\right), \\
& {\left[g_{n}^{-1} \widetilde{g} g_{0}, g_{0}^{-1} g_{1}, g_{1}^{-1} g_{2}, \ldots, g_{n-1}^{-1} g_{n}\right] \leftarrow \text { । }\left[\widetilde{g} ; g_{0} H, g_{1} H, \ldots, g_{n} H\right]}
\end{aligned}
$$

are well defined, mutually inverse, and they intertwine the coface, codegeneracy and cocyclic operators. Quite unexpected and remarkable fact is that this isomorphism of affine varieties which identifies orbits of different groups acting on different varieties identifies also their stabilizers, as is evident from the comparison of (3.17) and (3.18). This suggests that a more appropriate description should involve algebraic quotient stacks instead of varieties.

We note also that in (3.19), (3.20) and (3.21), the orbit $G / H$ can be replaced with an arbitrary left $G$-variety $X$. This produces a cocyclic object given by the coface operators

$$
\delta_{i}: G \backslash\left(\operatorname{ad}(G) \times X^{n+1}\right) \longrightarrow G \backslash\left(\operatorname{ad}(G) \times X^{n+2}\right),
$$




$$
\delta_{i}\left[\widetilde{g}, x_{0}, \ldots, x_{n}\right]=\left[\widetilde{g}, x_{0}, \ldots, x_{i}, x_{i}, \ldots, x_{n}\right],
$$

the codegeneracy operators

$$
\begin{aligned}
& \sigma_{i}: G \backslash\left(\operatorname{ad}(G) \times X^{n+1}\right) \longrightarrow G \backslash\left(\operatorname{ad}(G) \times X^{n}\right), \\
& \sigma_{i}\left[\widetilde{g}, x_{0}, \ldots, x_{n}\right]=\left[\widetilde{g}, x_{0}, \ldots, \widehat{x}_{i}, \ldots, x_{n}\right],
\end{aligned}
$$

and the cocyclic operator

$$
\begin{aligned}
& \tau_{n}: G \backslash\left(\operatorname{ad}(G) \times X^{n+1}\right) \longrightarrow G \backslash\left(\operatorname{ad}(G) \times X^{n+1}\right), \\
& \tau_{n}\left[\widetilde{g}, x_{0}, \ldots, x_{n}\right]=\left[\widetilde{g}, x_{1}, \ldots, x_{n}, \widetilde{g} x_{0}\right] .
\end{aligned}
$$

The periodic cyclic homology of its cyclic object of regular functions can be regarded as a $\mathbb{Z} / 2$ graded $G$-invariant cohomology of an affine $G$-variety $X$ with the equivariant system of coefficients in a vector space of regular functions on $\operatorname{ad}(G)$.

Such an ad-twisted cohomology has been introduced in [33], and is denoted by $\mathrm{H}_{\mathrm{ad}(G)}^{\bullet}(X)$. It is a module over the algebra $\mathrm{H}_{\mathrm{ad}(G)}^{\bullet}(\mathrm{pt})$ of ad-twisted cohomology of a one point $G$-variety. The latter is isomorphic to the algebra of regular class functions on $G$ put in the even degree [33]. For a trivial group $G$ and a smooth affine variety $X$ its ad-twisted cohomology coincides with its $\mathbb{Z} / 2$-graded de Rham cohomology $\mathrm{H}_{\mathrm{ad}(G)}^{\bullet}(X)=\mathrm{H}_{\mathrm{dR}}^{\bullet}(X)[33]$.

In [33] an isomorphism similar to (3.22), (3.23) (on the level of the Connes complex computing the cyclic homology) was used in order to construct a generalized character

$$
\operatorname{Rep}(G) \rightarrow \mathrm{H}_{\mathrm{ad}(G)}^{0}(G / H)
$$

transforming into the Chern character of an associated vector bundle under a Chern-Weil-like map, so called strong Cartan connection.

Moreover, for a finite $G$ we mention briefly the following relation between our construction and the Frobenius reciprocity. Let us denote by $X=H \backslash \operatorname{ad}(H)$ and $Y=G \backslash \operatorname{ad}(G)$ the affine (finite) varieties (over an algebraically closed field $k$ of characteristic not dividing the order of $G$ ) of conjugacy classes, and by $f: X \rightarrow Y$ the finite étale morphism induced by the containment $H<G$. We then have two maps between the algebras $A=\mathscr{O}(X)$ and $B=\mathscr{O}(Y)$ of regular functions. One is the algebra map $B \rightarrow A$ describing the morphism $f$, and the other is a $B$-linear map $\operatorname{Tr}_{f}: A \rightarrow B$ defined by means of the evaluation at points

$$
\left(\operatorname{Tr}_{f}(a)\right)(y)=\sum_{f(x)=y} a(x)
$$

Let $\chi \in A$ be a character of a representation of the subgroup $H$, and denote by $\chi \uparrow_{H}^{G} \in B$ the character of the induced representation. Our point is that the Frobenius reciprocity can be rewritten as

$$
\chi \uparrow{ }_{H}^{G}=\operatorname{Tr}_{f}(\chi),
$$

and the justification comes from the following canonical decomposition of $f$ according to our construction

$$
H \backslash(\operatorname{ad}(H) \times H / H) \stackrel{i}{\rightarrow} G \backslash(\operatorname{ad}(G) \times G / H) \stackrel{p}{\rightarrow} G \backslash(\operatorname{ad}(G) \times G / G) .
$$

Indeed, we have $\operatorname{Tr}_{f}=\operatorname{Tr}_{p} \circ \operatorname{Tr}_{i}$,

$$
A \stackrel{\operatorname{Tr}_{i}}{\longrightarrow} B^{\prime} \stackrel{\operatorname{Tr}_{p}}{\longrightarrow} B
$$


where $B^{\prime}=\mathscr{O}(G \backslash(\operatorname{ad}(G) \times G / H)), \operatorname{Tr}_{i}$ is an extension by zero outside the image of the closedopen immersion $i$, and $\operatorname{Tr}_{p}$ is the usual trace map along the fibers of the finite étale covering $p$. Since explicitly

$$
\operatorname{Tr}_{i}(a)([\widetilde{g}, g H])= \begin{cases}a\left(\left[g^{-1} \widetilde{g} g\right]\right), & \text { if } g^{-1} \widetilde{g} g \in H, \\ 0, & \text { otherwise }\end{cases}
$$

and

$$
\operatorname{Tr}_{p}\left(b^{\prime}\right)([\widetilde{g}])=\sum_{g H \in G / H} b^{\prime}([\widetilde{g}, g H])
$$

we have

$$
\operatorname{Tr}_{f}(a)([\widetilde{g}])=\sum_{g H \in G / H, g^{-1} \widetilde{g} g \in H} a\left(\left[g^{-1} \widetilde{g} g\right]\right),
$$

which applied to (3.27) gives the classical formulation of the Frobenius reciprocity

$$
\chi \uparrow \stackrel{G}{H}([\widetilde{g}])=\sum_{g H \in G / H, g^{-1} \widetilde{g} g \in H} \chi\left(\left[g^{-1} \widetilde{g} g\right]\right) .
$$

Note that in (3.27) the left hand side depends only on the representation theory, while the right hand side depends only on the geometry of the map $f$ of conjugation classes. This suggests that the Frobenius reciprocity in the form of (3.27) should be read in view of the perfect bilinear pairing provided by the evaluation of the algebra $\mathscr{O}(G \backslash \operatorname{ad}(G)$ ) of class functions (with a canonical basis consisting of the irreducible characters) against the center $\mathrm{Z}(k G)$ of the group algebra (with a canonical basis consisting of class sums corresponding to the conjugacy classes). The latter can be understood as a duality between the irreducible representations and the conjugacy classes.

Another interesting fact about the inverse isomorphism (3.23) is that it restricts to the collection of varieties of extended quotients in the sense of [1]. The extended quotient $G \backslash \backslash X$ of a (say left) $G$-variety $X$ has been defined in [1] as a usual quotient $G \backslash \widetilde{X}$ of a subvariety $\widetilde{X} \subseteq \operatorname{ad}(G) \times X$ of pairs $(\widetilde{g}, x)$ such that $\widetilde{g} x=x$ with respect to the left $G$-action $g(\widetilde{g}, x)=\left(g \widetilde{g} g^{-1}, g x\right)$. The extended quotient replaces the orbit by the variety of the conjugacy classes of the stabilizer, and plays a role in the local Langlands program [1]. For a finite $G$ it was used to define the orbifold cohomology [13] in terms of the inertia orbifold. Usually atributed to Chern-Ruan who explored its new orbifold cup-product, on the additive level it was in fact introduced earlier in a paper by Brylinski and Nistor, where the Chern-Ruan cohomology arises as the periodic cyclic homology of the convolution algebra of the groupoid associated to the orbifold [7, Corollary 5.10(ii)]. This is another evidence that cyclic homology of (quantum) stacks would be the most appropriate framework for considering our duality.

It is easy to see that the operators (3.24)-(3.26) restrict to the collection of extended quotients $G \backslash \backslash\left(X^{n+1}\right) \subseteq G \backslash\left(\operatorname{ad}(G) \times X^{n+1}\right)$ with respect to the diagonal $G$-action on the cartesian powers of a left $G$-variety $X$ making it a cocyclic variety. Note that the $G$-action on cartesian powers play a role in the problem of inertia factors in Clifford theory [34].

It is also easy to see that for the orbit $X=G / H$, the inverse isomorphism (3.23) restricts to $G \backslash \backslash\left((G / H)^{n+1}\right) \subseteq G \backslash\left(\operatorname{ad}(G) \times(G / H)^{n+1}\right)$, and transforms it into a subvariety of $G^{n+1} / H^{n+1}$ consisting of the orbits of $(n+1)$-tuples $\left(g_{0}, g_{1}, \ldots, g_{n}\right)$ such that $g_{i+1} \cdots g_{n} \cdot g_{0} \cdots g_{i} \in H$ for all $i=0, \ldots, n$. 


\section{Acknowledgements}

The authors would like to thank the anonymous referees for their constructive comments improving the paper. The paper was partially supported by the NCN grant 2011/01/B/ST1/06474. $\mathrm{S}$. Sütlü would like to thank his former $\mathrm{PhD}$ advisor B. Rangipour for drawing his attention to the homology of the coalgebra-Galois extensions, Institut des Hautes Études Scientifiques (IHES) for the hospitality provided during part of this work, and finally the organizers of the conference "From Poisson Brackets to Universal Quantum Symmetries", held at IMPAN, Warsaw, for the stimulating environment provided.

\section{References}

[1] Aubert A.M., Baum P., Plymen R., Solleveld M., Geometric structure in smooth dual and local Langlands conjecture, Jpn. J. Math. 9 (2014), 99-136, arXiv:1211.0180.

[2] Bergman G.M., Hausknecht A.O., Co-groups and co-rings in categories of associative rings, Mathematical Surveys and Monographs, Vol. 45, Amer. Math. Soc., Providence, RI, 1996.

[3] Bichon J., Hochschild homology of Hopf algebras and free Yetter-Drinfeld resolutions of the counit, Compos. Math. 149 (2013), 658-678, arXiv:1204.0687.

[4] Bonechi F., Ciccoli N., Dąbrowski L., Tarlini M., Bijectivity of the canonical map for the non-commutative instanton bundle, J. Geom. Phys. 51 (2004), 71-81, math.QA/0306114.

[5] Bonechi F., Ciccoli N., Tarlini M., Noncommutative instantons on the 4-sphere from quantum groups, Comm. Math. Phys. 226 (2002), 419-432, math.QA/0012236.

[6] Brown K.A., Zhang J.J., Dualising complexes and twisted Hochschild (co)homology for Noetherian Hopf algebras, J. Algebra 320 (2008), 1814-1850, math.RA/0603732.

[7] Brylinski J.L., Nistor V., Cyclic cohomology of étale groupoids, K-Theory 8 (1994), 341-365.

[8] Brzeziński T., Quantum homogeneous spaces as quantum quotient spaces, J. Math. Phys. 37 (1996), 23882399, q-alg/9509015.

[9] Brzeziński T., Quantum homogeneous spaces and coalgebra bundles, Rep. Math. Phys. 40 (1997), 179-185, q-alg/9704015.

[10] Brzeziński T., Hajac P.M., Coalgebra extensions and algebra coextensions of Galois type, Comm. Algebra 27 (1999), 1347-1367, q-alg/9708010.

[11] Brzeziński T., Hajac P.M., Galois-type extensions and equivariant projectivity, arXiv:0901.0141.

[12] Cartan H., Eilenberg S., Homological algebra, Princeton University Press, Princeton, NJ, 1956.

[13] Chen W., Ruan Y., A new cohomology theory of orbifold, Comm. Math. Phys. 248 (2004), 1-31, math.AG/0004129.

[14] Collins B., Härtel J., Thom A., Homology of free quantum groups, C. R. Math. Acad. Sci. Paris 347 (2009), 271-276, arXiv:0903.1686.

[15] Connes A., Cohomologie cyclique et foncteurs $\mathrm{Ext}^{n}$, C. R. Acad. Sci. Paris Sér. I Math. 296 (1983), 953-958.

[16] Connes A., Moscovici H., Hopf algebras, cyclic cohomology and the transverse index theorem, Comm. Math. Phys. 198 (1998), 199-246, math.DG/9806109.

[17] Connes A., Moscovici H., Cyclic cohomology and Hopf algebras, Lett. Math. Phys. 48 (1999), 97-108, math.QA/9904154.

[18] Dijkhuizen M.S., Koornwinder T.H., Quantum homogeneous spaces, duality and quantum 2-spheres, Geom. Dedicata 52 (1994), 291-315.

[19] Eckmann B., Cyclic homology of groups and the Bass conjecture, Comment. Math. Helv. 61 (1986), 193-202.

[20] Feng P., Tsygan B., Hochschild and cyclic homology of quantum groups, Comm. Math. Phys. 140 (1991), 481-521.

[21] Getzler E., Cartan homotopy formulas and the Gauss-Manin connection in cyclic homology, in Quantum Deformations of Algebras and their Representations (Ramat-Gan, 1991/1992; Rehovot, 1991/1992), Israel Math. Conf. Proc., Vol. 7, Bar-Ilan University, Ramat Gan, 1993, 65-78.

[22] Ginzburg V., Kumar S., Cohomology of quantum groups at roots of unity, Duke Math. J. 69 (1993), 179-198. 
[23] Hadfield T., Krähmer U., Twisted homology of quantum SL(2), K-Theory 34 (2005), 327-360, math.QA/0405249.

[24] Hajac P.M., Khalkhali M., Rangipour B., Sommerhäuser Y., Hopf-cyclic homology and cohomology with coefficients, C. R. Math. Acad. Sci. Paris 338 (2004), 667-672, math.KT/0306288.

[25] Hajac P.M., Khalkhali M., Rangipour B., Sommerhäuser Y., Stable anti-Yetter-Drinfeld modules, C. R. Math. Acad. Sci. Paris 338 (2004), 587-590, math.QA/0405005.

[26] Hochschild G., Kostant B., Rosenberg A., Differential forms on regular affine algebras, Trans. Amer. Math. Soc. 102 (1962), 383-408.

[27] Jara P., Ştefan D., Hopf-cyclic homology and relative cyclic homology of Hopf-Galois extensions, Proc. London Math. Soc. 93 (2006), 138-174.

[28] Kadison L., A relative cyclic cohomology theory useful for computations, C. R. Acad. Sci. Paris Sér. I Math. 308 (1989), 569-573.

[29] Kadison L., Cyclic homology of triangular matrix algebras, in Topology Hawaii (Honolulu, HI, 1990), World Sci. Publ., River Edge, NJ, 1992, 137-148.

[30] Kadison L., Hopf subalgebras and tensor powers of generalized permutation modules, J. Pure Appl. Algebra 218 (2014), 367-380, arXiv:1210.3178.

[31] Khalkhali M., Rangipour B., A note on cyclic duality and Hopf algebras, Comm. Algebra 33 (2005), 763-773, math.KT/0310088.

[32] Lu J.H., Moment maps at the quantum level, Comm. Math. Phys. 157 (1993), 389-404.

[33] Maszczyk T., Feynman integral, Chern character and duality, in preparation.

[34] Pahlings H., Plesken W., Group actions on Cartesian powers with applications to representation theory, J. Reine Angew. Math. 380 (1987), 178-195.

[35] Podleś P., Quantum spheres, Lett. Math. Phys. 14 (1987), 193-202.

[36] Schafer J.A., Relative cyclic homology and the Bass conjecture, Comment. Math. Helv. 67 (1992), 214-225.

[37] Schauenburg P., Galois correspondences for Hopf bi-Galois extensions, J. Algebra 201 (1998), 53-70.

[38] Schneider H.J., Some remarks on exact sequences of quantum groups, Comm. Algebra 21 (1993), 3337-3357.

[39] Takeuchi M., A correspondence between Hopf ideals and sub-Hopf algebras, Manuscripta Math. 7 (1972), 251-270.

[40] Van Oystaeyen F., Zhang Y., Galois-type correspondences for Hopf-Galois extensions, K-Theory 8 (1994), $257-269$. 